\title{
Lytic polysaccharide monooxygenases from Myceliophthora thermophila C1 differ in substrate preference and reducing agent specificity
}

\author{
Matthias Frommhagen ${ }^{1}$, Martijn J. Koetsier ${ }^{2}$, Adrie H. Westphal ${ }^{3}$, Jaap Visser ${ }^{4}$, Sandra W. A. Hinz ${ }^{2}$, \\ Jean-Paul Vincken ${ }^{1}$, Willem J. H. van Berkel ${ }^{3}$, Mirjam A. Kabel ${ }^{1}$ and Harry Gruppen ${ }^{\text {* }}$
}

\begin{abstract}
Background: Lytic polysaccharide monooxgygenases (LPMOs) are known to boost the hydrolytic breakdown of lignocellulosic biomass, especially cellulose, due to their oxidative mechanism. For their activity, LPMOs require an electron donor for reducing the divalent copper cofactor. LPMO activities are mainly investigated with ascorbic acid as a reducing agent, but little is known about the effect of plant-derived reducing agents on LPMOs activity.

Results: Here, we show that three LPMOs from the fungus Myceliophthora thermophila C1, MtLPMO9A, MtLPMO9B and MtLPMO9C, differ in their substrate preference, C1-/C4-regioselectivity and reducing agent specificity. MtLPMO9A generated C1- and C4-oxidized, MtLPMO9B C1-oxidized and MtLPMO9C C4-oxidized gluco-oligosaccharides from cellulose. The recently published MtLPMO9A oxidized, next to cellulose, xylan, $\beta-(1 \rightarrow 3,1 \rightarrow 4)$-glucan and xyloglucan. In addition, MtLPMO9C oxidized, to a minor extent, xyloglucan and $\beta-(1 \rightarrow 3,1 \rightarrow 4)$-glucan from oat spelt at the C4 position. In total, 34 reducing agents, mainly plant-derived flavonoids and lignin-building blocks, were studied for their ability to promote LPMO activity. Reducing agents with a 1,2-benzenediol or 1,2,3-benzenetriol moiety gave the highest release of oxidized and non-oxidized gluco-oligosaccharides from cellulose for all three MtLPMOs. Low activities toward cellulose were observed in the presence of monophenols and sulfur-containing compounds.

Conclusions: Several of the most powerful LPMO reducing agents of this study serve as lignin building blocks or protective flavonoids in plant biomass. Our findings support the hypothesis that LPMOs do not only vary in their C1-/ C4-regioselectivity and substrate specificity, but also in their reducing agent specificity. This work strongly supports the idea that the activity of LPMOs toward lignocellulosic biomass does not only depend on the ability to degrade plant polysaccharides like cellulose, but also on their specificity toward plant-derived reducing agents in situ.
\end{abstract}

Keywords: Electron donor, Phenolics, Flavonoids, Lignin, Glucan, Xylan

\section{Background}

Plant biomass utilization is considered to be a green approach for the production of renewable biofuels and biochemicals. Here, current developments aim at the effective degradation of the plant biomass polysaccharides, mostly embedded in a lignocellulosic complex, into

\footnotetext{
*Correspondence: harry.gruppen@wur.nl

1 Laboratory of Food Chemistry, Wageningen University, Bornse Weilanden 9, 6708 WG Wageningen, The Netherlands

Full list of author information is available at the end of the article
}

monosaccharides using enzyme cocktails. These commercial enzyme preparations usually originate from fungi such as Aspergillus and Trichoderma strains. Alternatively, the ascomycete Myceliophthora thermophila $\mathrm{C} 1$ is used to produce plant polysaccharide-degrading enzymes [1-3].

Lignocellulosic plant biomass is composed of the aromatic heteropolymer lignin and polysaccharides, such as hemicellulose and cellulose. In this research, we focus on the degradation of the highly recalcitrant polysaccharide 
cellulose. Cellulose is a homogenous polymer consisting of $\beta$ - $(1 \rightarrow 4)$-linked glucosyl chains. The interactions of these glucosyl chains via hydrogen bondings and van der Waal forces lead to the formation of crystalline cellulose regions [4]. These crystalline regions are difficult to access for most of the known hydrolytic cellulases listed in the Carbohydrate-Active enZyme (CAZy, [5]) database. The recently discovered lytic polysaccharide monooxygenases (LPMOs) are able to improve the hydrolytic breakdown of crystalline cellulose regions by their oxidative mechanisms $[6,7]$. It has also been shown that certain LPMOs oxidize chitin, hemicellulosic glucan, soluble cellodextrins, xylan or starch $[2,8-12]$. LPMOs are classified as "auxiliary activities" (AA) and are divided, based on their sequence similarity, into four CAZy subgroups: AA9, AA10, AA11 and AA13 [5].

The AA9 classified fungal LPMOs exhibit the oxidative cleavage of cellulose-yielding products with either the C1- (lactones) or C4- (ketoaldoses) position oxidized, or mixtures of the two $[6,13-15]$. The oxidative cleavage of substrates by LPMOs requires a divalent copper ion in the active site in addition to molecular oxygen and a reducing agent to oxidize one $\beta-(1 \rightarrow 4)$-linkage between the glucosyl residues [15].

Most studies on the activity of LPMOs published so far have been carried out with ascorbic acid as reducing agent $[2,6,10,16,17]$. Besides ascorbic acid, other small molecular weight reducing agents have been described to donate electrons to LPMOs, such as hydroquinone, catechin, and gallic acid and the macromolecule lignin $[9,13,14,18-20]$. Interestingly, it has been shown that reducing agents, especially the plant-derived diphenols, can be regenerated by GMC oxidoreductases [20]. Furthermore, flavocytochrome-dependent cellobiose dehydrogenases (CDHs) are reported to provide LPMOs with electrons and, more recently, light-excited photosynthetic pigments [21-23]. Literature is, however, ambiguous whether the type of reducing agent also influences the LPMO activity. On the one hand, it has been shown that the addition of different reducing agents influences the amount of released oxidized and non-oxidized glucooligosaccharides from PASC (phosphoric acid swollen cellulose) incubated with LPMO $[19,20]$. On the other hand, the addition of three different reducing agents to PASC incubated with PcGH61D did not affect the amounts of products released [7]. Therefore, it remains unknown whether different LPMOs share the same preference for the same type of reducing agent. In addition, for fungi it has been shown that polysaccharide sources used for fungal growth influence the expression of LPMO encoding genes [3]. Hence, we hypothesize that different LPMOs from $M$. thermophila C1 do not only vary in their C1-/C4-regioselectivity and substrate specificity, but also in their reducing agent specificity [3]. Therefore, we investigated the effect of 34 different reducing agents, in particular plant-derived reducing agents, such as flavonoids or lignin building blocks, on the cellulosedegrading activity of three LPMOs from $M$. thermophila C1. One of these LPMOs is MtLPMO9A, which has been shown to oxidize cellulose at the $\mathrm{C} 1$ - and $\mathrm{C} 4$ position [2]. For the two other LPMOs, $M t \mathrm{LPMO} 9 \mathrm{~B}$ and $M t \mathrm{LP}-$ MO9C, we also characterized the C1-/C4-regioselectivity and substrate specificity. Based on their chemical structure, all reducing agents were classified into five groups, partly reflecting their effect on the LPMO activity.

\section{Results}

\section{Purification of MtLPMO9B and MtLPMO9C}

$M t L P M O 9 B$ and $M t$ LPMO9C were produced in the homologous host $M$. thermophila $\mathrm{C} 1$ and purified from the culture broth supernatant in either three (MtLPMO9B) or four (MtLPMO9C) steps. Both final $M t L P-$ MO9B and $M t$ LPMO9C preparations showed single bands on SDS-PAGE (Additional file 1) with apparent subunit molecular masses of 32 and $25 \mathrm{kDa}$, respectively. The purified $M t \mathrm{LPMO} 9 \mathrm{~B}$ and $M t \mathrm{LPMO}$ C preparations were further analyzed by LC/UV/ESI-MS. The weighted average masses of $M t \mathrm{LPMO} 9 \mathrm{~B}$ and $M t \mathrm{LPMO} C \mathrm{C}$ were 32,765 and $24,640 \mathrm{Da}$, respectively. These values are somewhat higher than the theoretical molecular masses (30.6 and $23.5 \mathrm{kDa}$, respectively) calculated from the respective amino acid sequences. However, ESI-MS spectra $(m / z$ values $)$ of $M t$ LPMO9B and $M t$ LPMO9C showed that both LPMOs were glycosylated by hexoses. In particular, up to 13 and 5 glycosyl units were attached to $M t \mathrm{LPMO} 9 \mathrm{~B}$ and $M t \mathrm{LPMO}$ C, respectively (Additional file 2).

\section{The mode of action of MtLPMO9B and MtLPMO9C on amorphous cellulose}

The activities of the pure $M t$ LPMO9B and $M t$ LPMO9C enzymes were tested on regenerated amorphous cellulose (RAC), in both the presence and absence of ascorbic acid. Incubation of RAC in the presence and absence of ascorbic acid without LPMO addition did not cause autooxidation of RAC, since no non-oxidized or oxidized gluco-oligosaccharides were detected using HPAEC and MALDI-TOF MS. The products released from RAC, after incubation with $M t \mathrm{LPMO} 9 \mathrm{~B}$ or $M t \mathrm{LPMO} 9 \mathrm{C}$, are shown in Fig. 1. Besides non-oxidized gluco-oligosaccharides $\left(\mathrm{GlcOS}_{\mathrm{n}}\right)$, only C1-oxidized gluco-oligosaccharides $\left(\operatorname{GlcOS}_{n}^{\#}\right)$ were formed upon incubation of RAC with $M t$ LPMO9B in the presence of ascorbic acid (Fig. 1b). In contrast, RAC incubated with $M t$ LPMO9C in the presence of ascorbic acid yielded only C4-oxidized gluco-oligosaccharides $\left(\mathrm{GlcOS}_{\mathrm{n}}^{*}\right)$, besides non-oxidized 


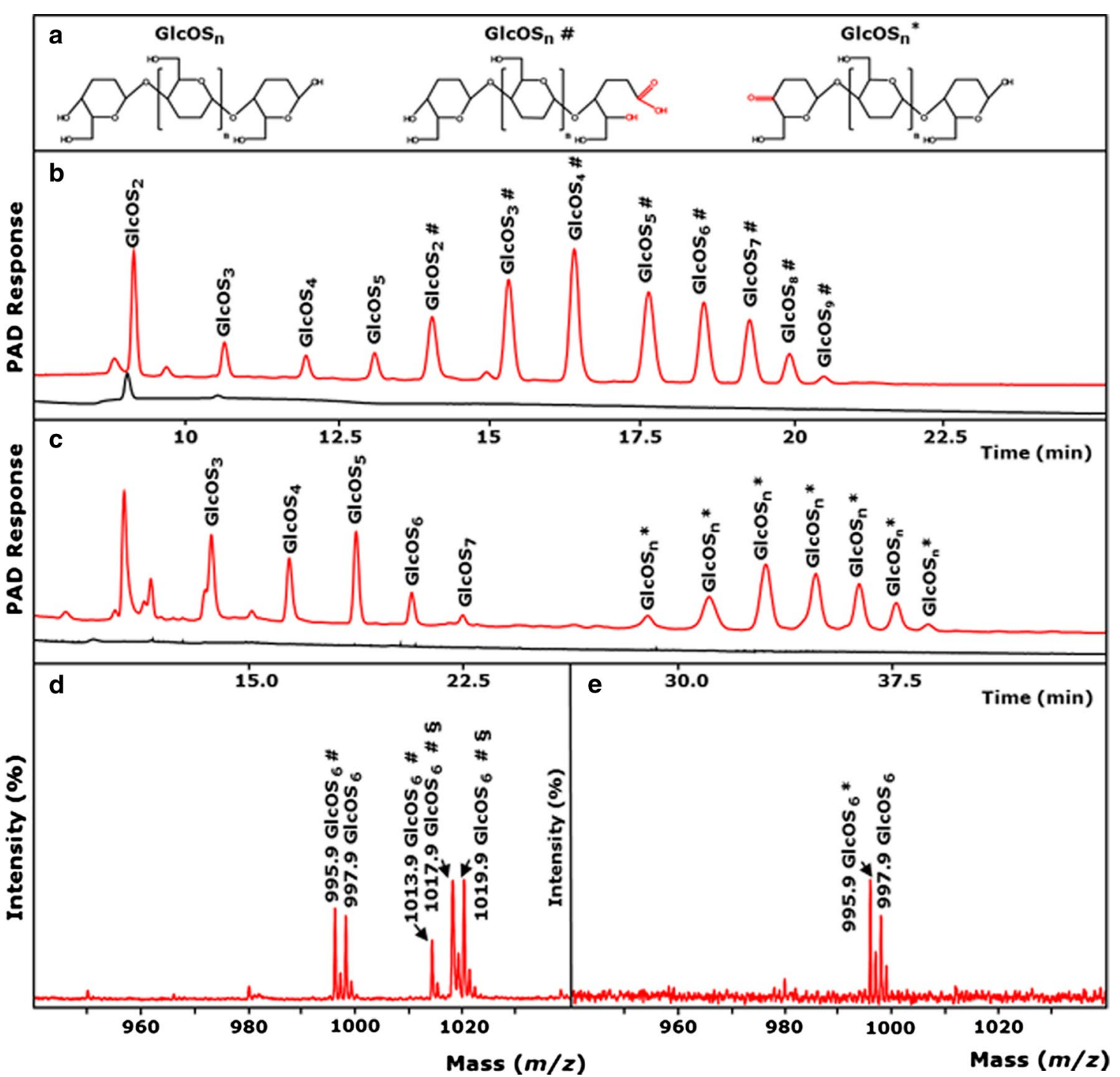

Fig. 1 Activity of MtLPMO9B and MtLPMO9C on regenerated amorphous cellulose. a Structure and nomenclature of released non-oxidized and C1- and C4-oxidized gluco-oligosaccharides (GlcOS $n^{\prime}$ GlcOS $_{n^{\prime}}^{*} \mathrm{GlCOS}_{n,}^{*}$ respectively). HPAEC elution pattern of regenerated amorphous cellulose (RAC; $2 \mathrm{mg} \times \mathrm{mL}^{-1}$ ) incubated with b MtLPMO9B (10 $\mathrm{mg} \times \mathrm{g}^{-1}$ substrate) and c MtLPMO9C (10 mg $\times \mathrm{g}^{-1}$ substrate), in the presence (1 mM, red line) and absence of ascorbic acid (black line). A different gradient was used for the separation of $\mathbf{b}$ C1- and $\mathbf{c}$ C4-oxidized gluco-oligosaccharides (See "Methods"). The (4-oxidized gluco-oligosaccharides are known to be unstable under the alkaline conditions present during HPAEC analysis and undergo further derivatization to gem-diols, which are actually annotated as C4-oxidized gluco-oligosaccharides (GlcOS ${ }_{n}^{*}$ [34]. MALDI-TOF mass spectrum ( $\mathrm{m} / \mathrm{z}$ values) of RAC incubated with $\mathbf{d}$ MtLPMO9B or $\mathbf{e}$ MtLPMO9C, in the presence of ascorbic acid. d Double $\mathrm{Li}^{-}$adducts of C1-oxidized glucooligosaccharides are marked with section symbol. d Oxidation of the C1-carbon atom results in the formation non-oxidized gluco-oligosaccharides $\left(G_{C O S}\right)$ and C1-oxidized gluco-oligosaccharides present as a $\delta$-lactone (-2 Da, marked as GlcOS $)$. Lactones are unstable and convert to aldonic acids by the addition of water, leading to a $16 \mathrm{Da}$ higher mass compared to the non-oxidized gluco-oligosaccharide (+16 Da, marked as GlcOS ${ }_{n}^{\#}$ ). Double $\mathrm{Li}^{-}$adducts of C1-oxidized gluco-oligosaccharides are marked with section symbol (GlcOS ${ }_{6}^{\#} \$, 1019.9$ and 1017.9 Da, probably due to double oxidation). e Oxidation of the (4-carbon atom results in the formation of non-oxidized gluco-oligosaccharides (GlcOS $)$ and C4-oxidized glucooligosaccharides present as ketoaldoses (-2 Da, marked as GlcOS ${ }_{n}^{*}$. No gem-diols were formed. For more information see "Methods"

gluco-oligosaccharides $\left(\mathrm{GlcOS}_{\mathrm{n}}\right)$ (Fig. 1c). For both enzymes, neither oxidized nor non-oxidized gluco-oligosaccharides were released from RAC in the absence of ascorbic acid, which showed that cellulolytic activity was completely absent (Fig. 1b, c). MALDI-TOF MS confirmed the annotation of the HPAEC eluted glucooligosaccharides (Fig. 1d, e; Additional file 3a, b). For example, incubation of RAC with $M t L P M O 9 B$ released non-oxidized cellohexaose with a mass of $997 \mathrm{Da}$ (lithium adduct) and C1-oxidized cellohexaose appeared 
either as a lactone (995 Da) or as the corresponding aldonic acid (1013 Da) (Fig. 1d). Masses of lactonic acid double substituted with lithium were also detected (1019 and $1017 \mathrm{Da}$, probably due to double oxidation). In contrast, RAC incubated with $M t$ LPMO9C only formed the C4-oxidized ketoaldose (995 Da) of cellohexaose and non-oxidized cellohexaose (997 Da, Fig. 1e). In summary, $M t$ LPMO9B oxidizes cellulose at the $\mathrm{C} 1-$ and $M t \mathrm{LP}-$ $\mathrm{MO} 9 \mathrm{C}$ at the C4-position, while the previously characterized MtLPMO9A oxidizes cellulose at the $\mathrm{C} 1$ - and C4-positon [2].

\section{Structure-based sequence alignment of MtLPMO9A, MtLPMO9B and MtLPMO9C and structural models of MtLPMO9B and MtLPMO9C}

Structure-based sequence alignments of MtLPMO9A, $M t \mathrm{LPMO} 9 \mathrm{~B}$ and $M t \mathrm{LPMO} 9 \mathrm{C}$ were constructed, based on the sequences of a C1-(PcLPMO9D; PDB ID code 4B5Q), C4-(NcLPMO9C; PDB ID code 4D7U) and C1- and C4-(TaLPMO9A; PDB ID code 3ZUD) oxidizing LPMO as presented by Borisova et al. (Fig. 2) [10, 14, 24, 25]. The three-dimensional structural models of $M t \mathrm{LPMO} 9 \mathrm{~B}$ and $M t$ LPMO9C (Fig. 3a, b) were generated based on the available structure of $\mathrm{NcLPMO}$ C from Neurospora crassa [25] (Protein Data Bank entry: 4D7U). MtLPMO9B is, unlike $M t \mathrm{LPMO}$ C and the previously published $M t \mathrm{LP}-$ MO9A [2], linked at the C-terminal end to a carbohydrate-binding module 1 (CBM1), which is not presented in the structural model of $M t \mathrm{LPMO}$. The sequences used for the structural-based alignments and models do not include the signal peptides and start from the $\mathrm{N}$-terminal histidine (His1). The overall sequence identity of $M t \mathrm{LPMO} 9 \mathrm{~A}, M t \mathrm{LPMO} 9 \mathrm{~B}$ and $M t \mathrm{LPMO}$ C ranges from 41 to $46 \%$. All three $M t$ LPMOs share the LPMO typical $\beta$-sheet core, but differ in their loop regions L2 (10-49), LS (114-128) and LC (176-226) that are involved in shaping the substrate-binding surface (Fig. 2) [2, 25, 26]. Interestingly, $M t \mathrm{LPMO} 9 \mathrm{C}$ and partly $M t \mathrm{LPMO}$ B $\mathrm{B}$ contain an insertion, which forms the L3 (64-78) loop region (Fig. 2). This L3 region is a typical structural characteristic of C4-oxidizing AA9 LPMOs [25-27]. Based on the model, $M t$ LPMO9B contains distal from the coordinated copper sphere an additional loop (Gly115-Asn121), which is not present in $M t$ LPMO9A and MtLPMO9C (Fig. 2a, b). The copper ion in $M t$ LPMO9A, $M t$ LPMO9B and $M t$ LPMO9C is coordinated by His1-His68-Tyr153, His1-His79-Tyr170 and His1-His84-Tyr166, respectively (Figs. 2, 3) [2]. All three $M t$ LPMOs share two putative disulfide bridges, presumably involved in stabilizing the different loops regions such as Cys126-Cys208 (LS-LC) for MtLPMO9A and Cys28-Cys178 (L2-LC) for MtLPMO9B (Fig. 3) [2]. In addition, it is likely that the neighboring Cys 18 and Cys49 of MtLPMO9B form a second disulfide bond
(L2-L2) (Fig. 3). The putative disulfide bridges of $M t \mathrm{LP}-$ MO9C between Cys39-Cys169 and Cys139-Cys221 are not expected to be involved in interlinking any of the four described loop regions (Fig. 2). All three MtLPMOs share the presence of several aromatic amino acid residues in the substrate-binding surface, which were formerly used for classifying the AA9s into subgroups (Figs. 2, 3) [2, 28, 29].

\section{Activity of MtLPMO9B and MtLPMO9C with various soluble and insoluble polysaccharides}

Various soluble and insoluble substrates were incubated with purified $M t \mathrm{LPMO} 9 \mathrm{~B}$ or $M t \mathrm{LPMO} 9 \mathrm{C}$ in the presence of ascorbic acid. The overview of the activities observed is presented in Table 1, which, for comparison, includes the activities of the previously published $M t$ LPMO9A [2]. Of all substrates tested, $M t$ LPMO9B showed only oxidative activity toward RAC, releasing C1-oxidized and non-oxidized gluco-oligosaccharides (Fig. 1b). MtLPMO9C oxidized RAC (Fig. 1c) releasing C4-oxidized and non-oxidized gluco-oligosaccharides and showed also activity toward $\beta-(1 \rightarrow 3,1 \rightarrow 4)$-glucan from oat spelt or xyloglucan from tamarind seed under the formation of C4-oxidized gluco-oligosaccharides and substituted C4-oxidized gluco-oligosaccharides (Additional files $4,5,6)$. No oxidized gluco-oligosaccharides were released from $\beta$ - $(1 \rightarrow 3,1 \rightarrow 4)$-glucan from barley incubated with $M t \mathrm{LPMO} 9 \mathrm{~B}$ or $M t \mathrm{LPMO} 9 \mathrm{C}$.

$M t$ LPMO9A has been described to cleave xylan associated with cellulose [2] forming oxidized xylo-oligosaccharides and oxidized gluco-oligosaccharides (Table 1). Hence, $M t \mathrm{LPMO} 9 \mathrm{~B}$ and $M t \mathrm{LPMO} C \mathrm{C}$ were also studied for their activity toward RAC-xylan mixtures, in particular, RAC mixed with either birchwood xylan, oat spelt xylan or wheat arabinoxylan, in the presence and absence of ascorbic acid (Table 1). No oxidized xylo-oligosaccharides were released by $M t \mathrm{LPMO} \mathrm{B}$ and $M t \mathrm{LPMO} 9 \mathrm{C}$, which discriminates these enzymes from $M t$ LPMO9A.

\section{Electron donor specificities of MtLPMO9A, MtLPMO9B and MtLPMO9C}

Based on the structural similarities of functional groups, the 34 reducing agents tested were classified into five groups (Fig. 4). Monophenols, like sinapic acid (no. 5), are classified as group I. Group II comprises compounds with a benzenediol moiety, which represent the ortho-isomer 1,2-benzenediol, the meta-isomer 1,3-benzenediol and the para-isomer 1,4-benzenediol (Fig. 4). Reducing agents with a 1,2,3-benzenetriol moiety are classified as group III. Reducing agents of group IV are sulfur-containing compounds, such as glutathione (no. 29) or L-cysteine (no. 30). Reducing agents of group V have neither a phenolic ring nor a sulfur atom. 


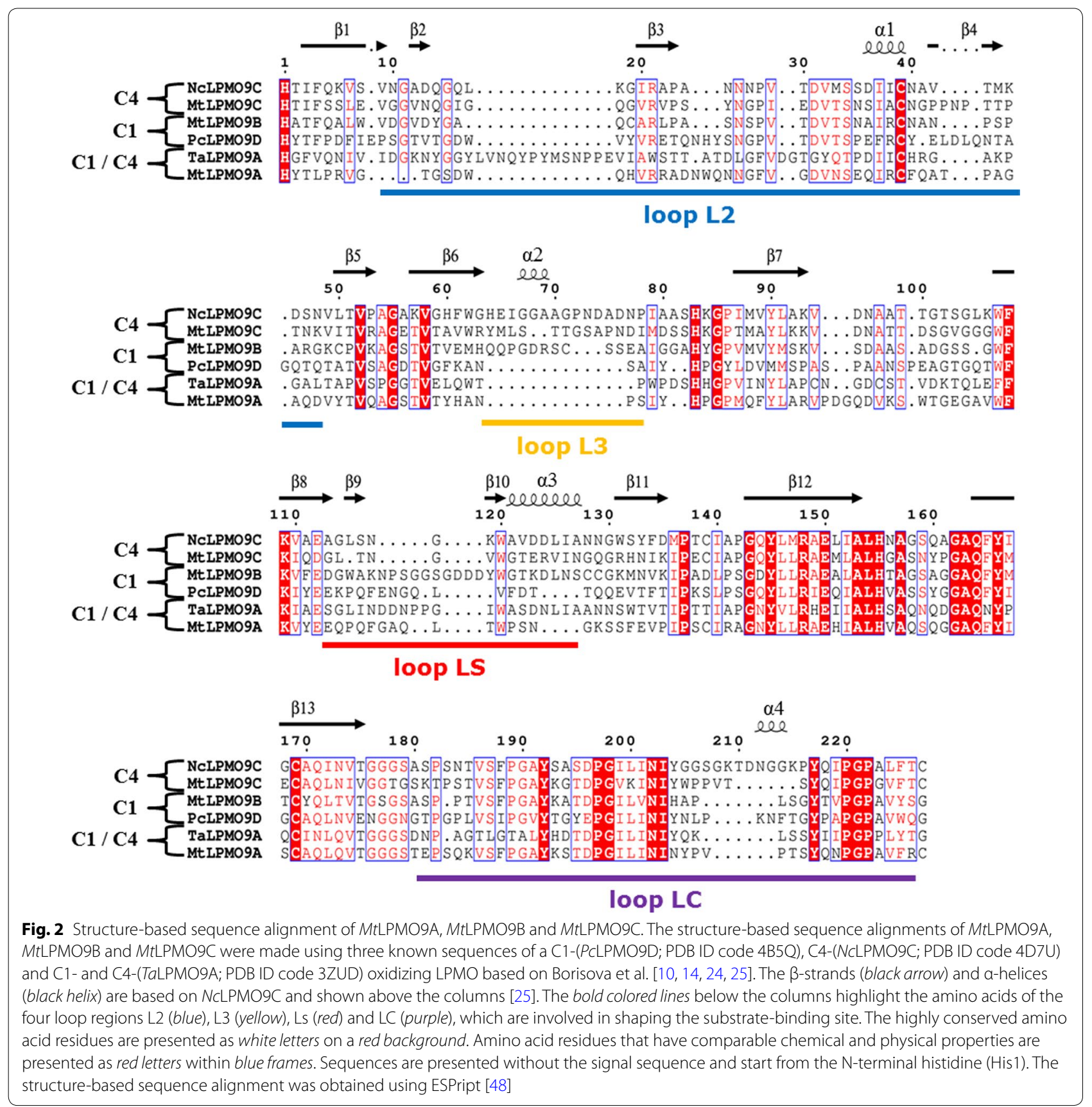

An overview of the effect of 34 different reducing agents with corresponding codes (no.) on the amounts of oxidized and non-oxidized gluco-oligosaccharides released from RAC incubated with $M t$ LPMO9A, $M t$ LPMO9B or $M t$ LPMO9C is presented in Table 2. First, we determined from the HPAEC profiles the amounts, expressed as peak areas, of $\mathrm{C} 1$-oxidized gluco-oligosaccharides, C4-oxidized gluco-oligosaccharides and non-oxidized gluco-oligosaccharides formed (Fig. 5). Subsequently, these amounts were presented as percentage of the corresponding amounts obtained by the same incubation, but with ascorbic acid (no. 33), which was set to $100 \%$ (Table 2). All 34 reducing agents were also incubated with RAC alone, which did not result in the release of oxidized or non-oxidized gluco-oligosaccharides, confirming the absence of auto-oxidation in the presence of the reducing agents only.

Fourteen reducing agents (Table 2, italics), including ascorbic acid, were able to donate electrons to all three $M t$ LPMOs enabling the oxidative cleavage of RAC. 

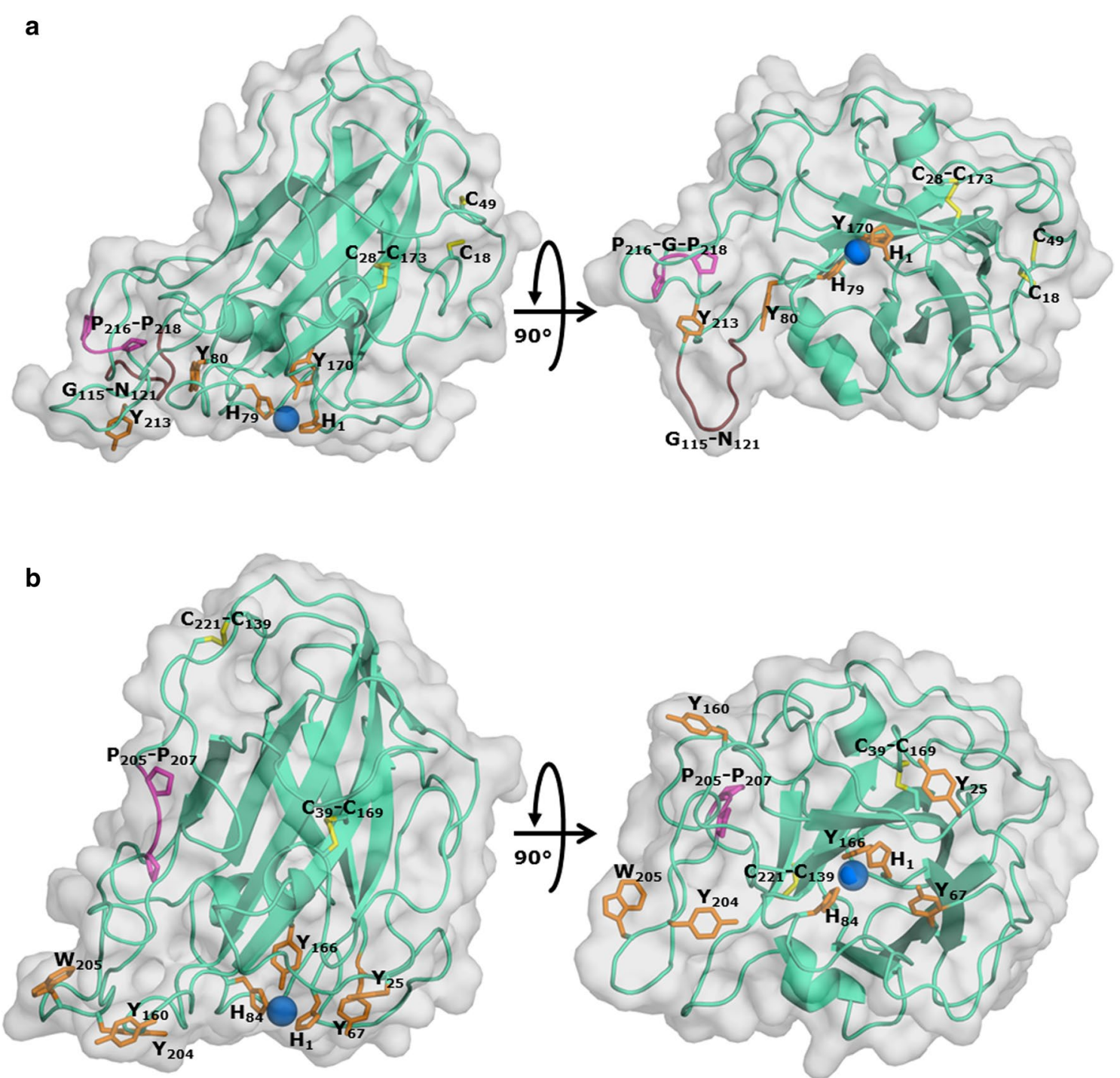

Fig. 3 Structural models of MtLPMO9B and MtLPMO9C. The structural model of a MtLPMO9B was generated based on the available structure of NCLPMO9C from Neurospora crassa [25] (PDB entry: 4D7U). The amino acid sequence identities of MtLPMO9B and MtLPMO9C compared to NCLPMO9C were 41 and $46 \%$, respectively. The copper ion (blue) is coordinated by His 1, His 79 and Tyr170 (orange). A disulfide bridge is located between Cys 28 and Cys 178 (yellow) and it is likely that the neighboring Cys18 and Cys49 form a second disulfide bridge. The structural model of b MtLPMO9C was, like MtLPMO9B, generated based on NCLPMO9C [25] (PDB entry: 4D7U). The copper ion (blue) is coordinated by His1, His84 and Tyr166 (orange). MtLPMO9C contains two disulfide bridges between Cys39 and Cys169, and Cys139 and Cys221 (yellow). The highly conserved Gly-Pro-Gly triad (magenta) of MtLPMO9B and MtLPMO9C is located between Pro216 and Pro218, and Pro205 and Pro207, respectively. MtLPMO9B contains, unlike MtLPMO9C, an additional loop between Gly-115 and Asn121 (brown)

Nevertheless, the LPMO activity, based on the amounts of oxidized gluco-oligosaccharides formed, varied per reducing agent (Fig. 5; Table 2). For all three $M t \mathrm{LP}$ MOs, ascorbic acid was one of the best electron donors (Table 2). Besides ascorbic acid, compounds bearing a 1,2-benzenediol moiety [3-methylcatechol (no. 14), 3,4-dihydroxyphenylalanine (no. 21)] or 1,2,3-benzenetriol moiety [gallic acid (no. 26), epigallocatechin-gallate (no. 27)] gave the highest formation of oxidized and non-oxidized gluco-oligosaccharides. Interestingly, dopamine (no. 21) turned out to be one of the best electron donors for $M t$ LPMO9A (93 \% of the activity compared to ascorbic acid), but was a less efficient electron donor for $M t \mathrm{LPMO} 9 \mathrm{~B}$ and $M t \mathrm{LPMO}$ C (46 and $6 \%$ compared to ascorbic acid, respectively). Some compounds bearing a 1,2-benzenediol moiety, for example quercetin or taxifolin (no. 23), and the benzenetriol tannic acid (no. 28) did not donate electrons to all three MtLPMOs. Out of 
Table 1 Oxidation of various polysaccharide substrates by MtLPMO9A, MtLPMO9B and MtLPMO9C

\begin{tabular}{|c|c|c|c|c|c|c|c|c|}
\hline \multirow[t]{3}{*}{ Substrate } & \multicolumn{8}{|c|}{ Occurrence of oxidation (upon addition of $1 \mathrm{mM}$ ascorbic acid) } \\
\hline & \multicolumn{2}{|c|}{ MtLPMO9A } & \multicolumn{2}{|c|}{ MtLPMO9B } & \multicolumn{2}{|c|}{ MtLPMO9C } & \multicolumn{2}{|c|}{ No enzyme } \\
\hline & $\mathrm{GlcOS}_{n}^{\# * \text { b }}$ & $\operatorname{xOS}_{n}^{\# *}{ }^{*} c$ & $\mathrm{GlcOS}_{n}^{\#}$ & $\operatorname{xOS}_{n}^{\#}$ & $\mathrm{GlcOS}_{n}{ }^{*}$ & $\operatorname{xos}_{n}{ }^{*}$ & $\mathrm{GlcOS}_{n}^{*}$ & $\operatorname{xos}_{n}^{*}$ \\
\hline \multicolumn{9}{|l|}{ Cellulose } \\
\hline RAC $^{\mathrm{d}}$ & + & + & + & - & + & - & - & - \\
\hline \multicolumn{9}{|l|}{ Hemicellulose } \\
\hline \multicolumn{9}{|l|}{ Glucan } \\
\hline Xyloglucan ${ }^{\mathrm{e}}$ & + & - & - & - & + & - & - & - \\
\hline$\beta$-Glucan barley ${ }^{e}$ & + & - & - & - & - & - & - & - \\
\hline$\beta$-Glucan oat spelt ${ }^{\mathrm{e}}$ & + & - & - & - & + & - & - & - \\
\hline \multicolumn{9}{|l|}{ Xylan } \\
\hline OSX $x^{f}$ & - & - & - & - & - & - & - & - \\
\hline$B i W X^{f}$ & - & - & - & - & - & - & - & - \\
\hline WAX & - & - & - & - & - & - & - & - \\
\hline \multicolumn{9}{|l|}{ Oligosaccharides } \\
\hline Gluco-oligosaccharides $^{9}$ & - & - & - & - & - & - & - & - \\
\hline Xylo-oligosaccharides ${ }^{g}$ & - & - & - & - & - & - & - & - \\
\hline \multicolumn{9}{|c|}{ RAC/Hemicellulose combination } \\
\hline$R A C+B i W X$ & + & + & + & - & + & - & - & - \\
\hline RAC + OSX & + & + & + & - & + & - & - & - \\
\hline RAC + WAX & + & - & + & - & + & - & - & - \\
\hline \multicolumn{9}{|c|}{ Oligosaccharides released and not released refers to + and - , respectively } \\
\hline \multicolumn{9}{|c|}{ a Data from Frommhagen et al. [2] } \\
\hline \multicolumn{9}{|c|}{${ }^{\mathrm{b}}$ Gluco-oligosaccharides oxidized at the $\mathrm{C} 1\left(\mathrm{GlcOS}_{n}^{\#}\right)$ or $\mathrm{C} 4$ position $\left(\mathrm{GlcOS}_{n}{ }^{*}\right)$} \\
\hline \multicolumn{9}{|c|}{ ' Xylo-oligosaccharides oxidized at the $\mathrm{C} 1\left(\mathrm{XOS}_{n}^{\#}\right)$ or $\mathrm{C} 4$ position $\left(\mathrm{XOS}_{n}{ }^{*}\right)$} \\
\hline \multicolumn{9}{|c|}{${ }^{d}$ Regenerated amorphous cellulose (RAC) } \\
\hline \multicolumn{9}{|c|}{ e Xyloglucan from tamarind seed, $\beta-(1 \rightarrow 3,1 \rightarrow 4)$-glucan from barley and $\beta$ - $(1 \rightarrow 3,1 \rightarrow 4)$-glucan from oat spelt } \\
\hline \multicolumn{9}{|c|}{ f Oat spelt xylan (OSX), birchwood xylan (BiWX), wheat arabinoxylan (WAX) } \\
\hline${ }^{g} \beta-(1 \rightarrow 4)$-linked gluco- and & r & egree of & & & & & & \\
\hline
\end{tabular}

seven monophenols, only sinapic acid (no. 5) acted as electron donor for all three MtLPMOs, but less efficient (4-23\%) compared to ascorbic acid (no. 33). A poor electron-donating capacity was also found for sulfur-containing compounds, such as reduced glutathione (no. 29) and L-cysteine (no. 30) (Table 2). Both thiol compounds have already been described as LPMO electron donors in literature $[7,30]$. In summary, the presence of different reducing agents strongly influences the release of oxidized and non-oxidized gluco-oligosaccharides from RAC for all three $M t$ LPMOs. Based on these findings, we conclude that $M t \mathrm{LPMO} 9 \mathrm{~A}, M t \mathrm{LPMO} 9 \mathrm{~B}$ and $M t \mathrm{LP}-$ MO9C differ in their specificity toward reducing agents.

\section{Discussion}

The recently discovered LPMOs play a crucial role in the enzymatic degradation of lignocellulosic plant biomass. Here, we purified the C1-oxidizing MtLPMO9B and C4-oxidizing $M t$ LPMO9C, two novel LPMOs from the filamentous fungus $M$. thermophila $\mathrm{C} 1$, and compared their catalytic properties with those of the $\mathrm{C} 1$ - and C4-oxidizing $M t$ LPMO9A [2].

\section{Protein mass}

The difference in molecular mass between $M t$ LPMO9B and $M t \mathrm{LPMO} 9 \mathrm{C}$ results from the CBM1 linked to $M t \mathrm{LP}$ MO9B. This CBM1 has a predicted mass, calculated from the amino acid sequence of $6811 \mathrm{Da}$. The weighted average mass of the purified MtLPMO9B linked to CBM1 and $M t$ LPMO9C enzymes (32.765 and $24.640 \mathrm{Da}$, respectively) differ slightly from the predicted masses, calculated from the amino acid sequences (30.633 and 23.449 Da, respectively). Both $M t \mathrm{LPMO} 9 \mathrm{~B}$ and $M t \mathrm{LPMO} 9 \mathrm{C}$ contain multiple glycosylations that were determined as hexoses based on LC/ESI-MS (Additional file 2). It is known that LPMOs can show glycosylation in the substrate-binding site, though the glycosylation could also appear at the serine/threonine containing linker between the carbohydrate-binding module (CBM), the C-terminal end of the LPMO or at the binding site of the CBM [29, 31, 32]. 


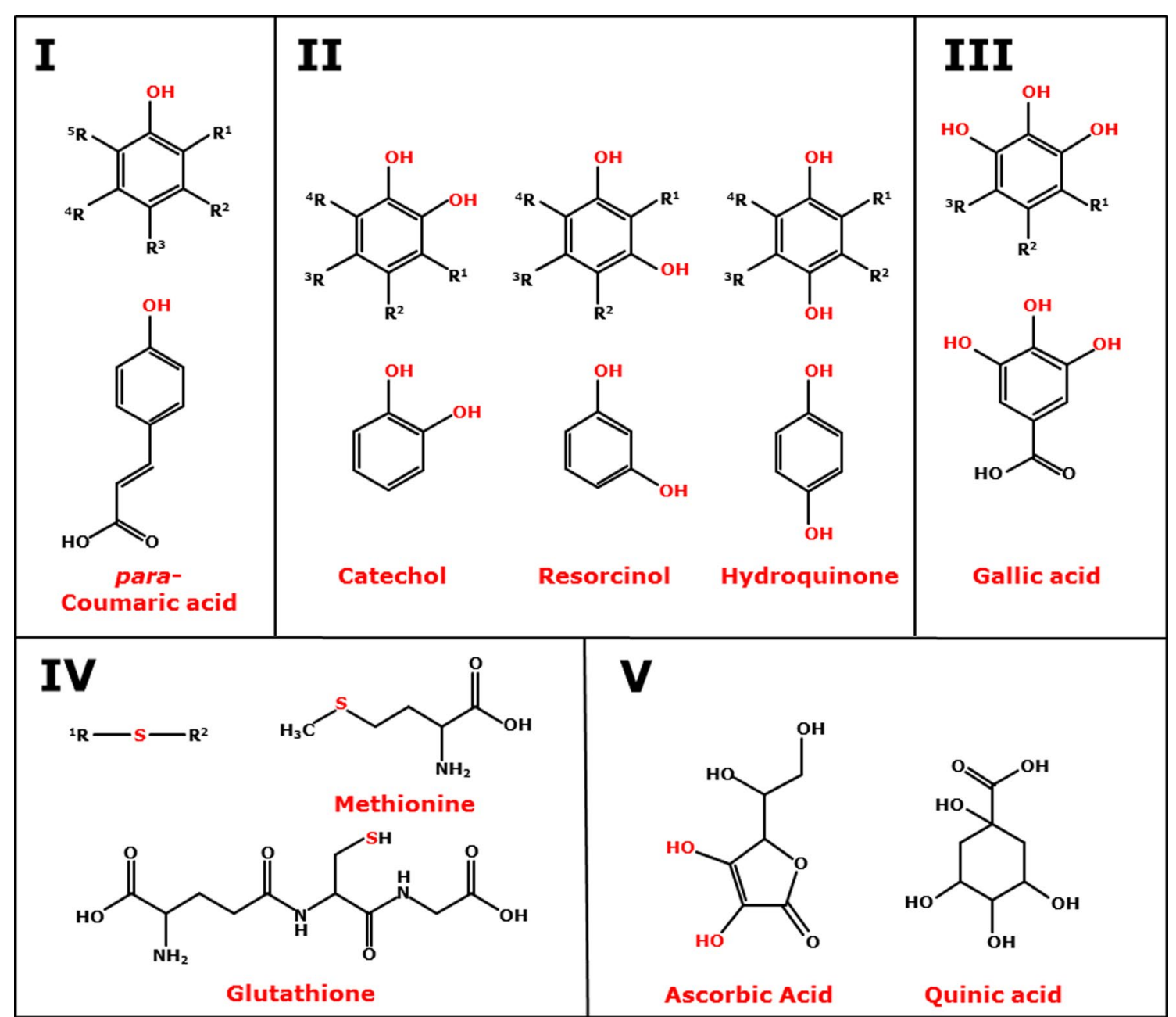

Fig. 4 Structural classification of reducing agents into five groups. Group I, monophenols. Group II, ortho-, meta- or para-isomers of compounds bearing a benzenediol moiety. Group III, reducing agents with a 1,2,3-benzenetriol moiety. Group IV, sulfur-containing compounds. Group $\mathbf{V}$, compounds with neither a phenolic ring nor a sulfur atom

\section{Activity on cellulose}

The total amount of released oxidized and non-oxidized gluco-oligosaccharides from RAC incubated with $M t$ LPMO9B is approximately three times higher (based on total AUC) compared to the released products from RAC by the $M t$ LPMO9A using ascorbic acid as a reducing agent (Fig. 5). We suggest that the CBM1 attached to $M t$ LPMO9B has a strong affinity to cellulose and supports the activity of $M t \mathrm{LPMO}$ B through the positioning of the MtLPMO9B to the cellulose. Our finding is supported by previous studies which showed that LPMOs linked to a CBM release more oxidized gluco-oligosaccharides from cellulose compared to LPMOs without a CBM [17, 33]. In addition, it has been hypothesized that glycosylation could affect the binding to cellulose, altering the enzyme activity [29]. So far, it has only been demonstrated for CBMs that the glycosylation of proteins with mannose in the planar face can increase the substrate-binding strength toward cellulose and not yet for LPMOs [29, 32]. However, it has been reported that the removal of a CBM1 linked to NCLPMO9C did not show any effect on the degradation rate of amorphous cellulose [25]. Still, a direct comparison of all three LPMOs regarding their activity on cellulose would require the absolute quantification of the released C1- and C4-oxidized glucooligosaccharides, which is so far not possible due to the lack of standards. Especially, the quantification of C4-oxidized products has its limits due to the instability of these compounds under alkaline conditions during HPAEC analysis [34]. The release of high amounts of non-oxidized gluco-oligosaccharides observed in the HPAEC spectra is likely to derive, to a certain extent, from the 


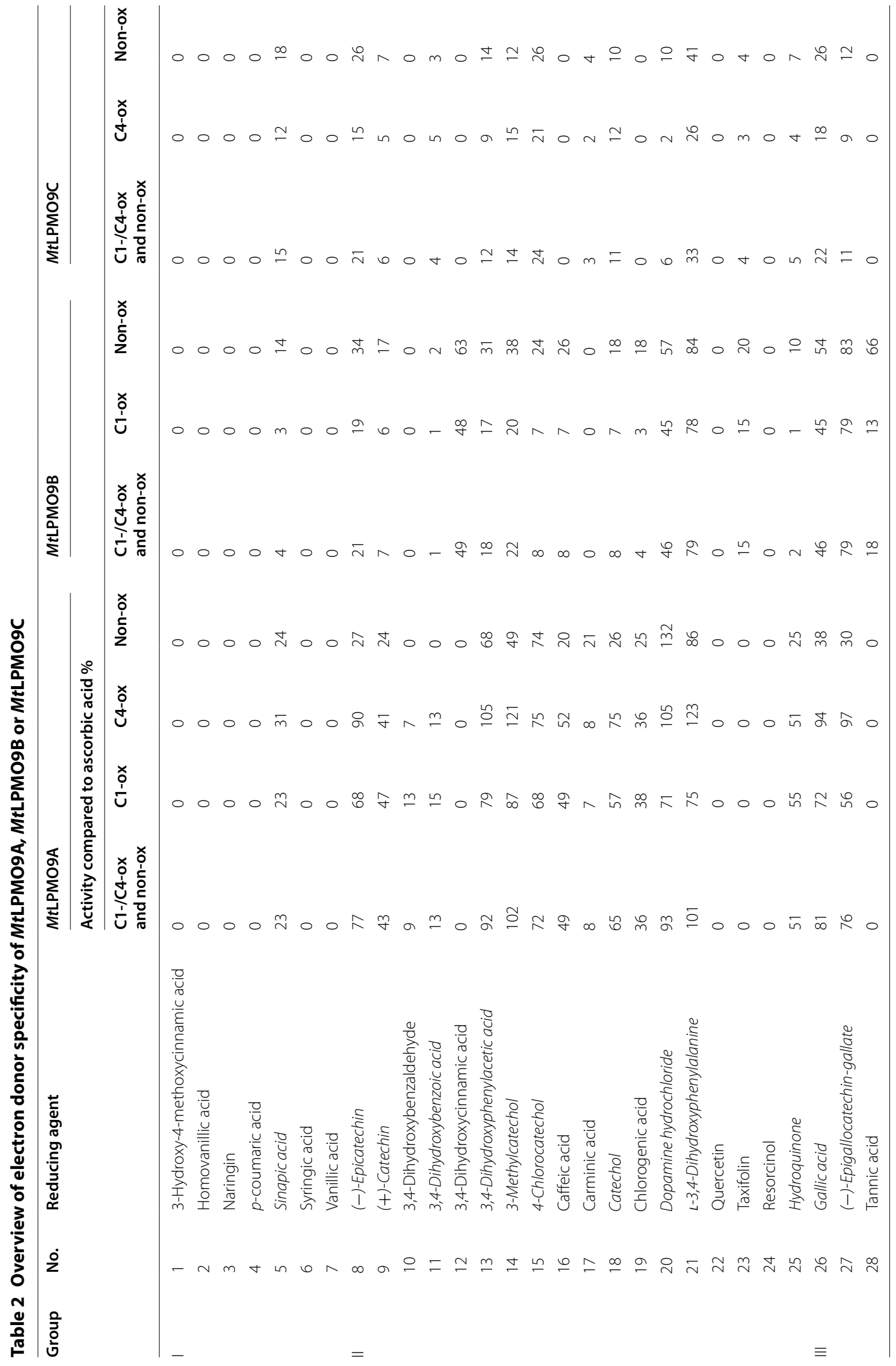




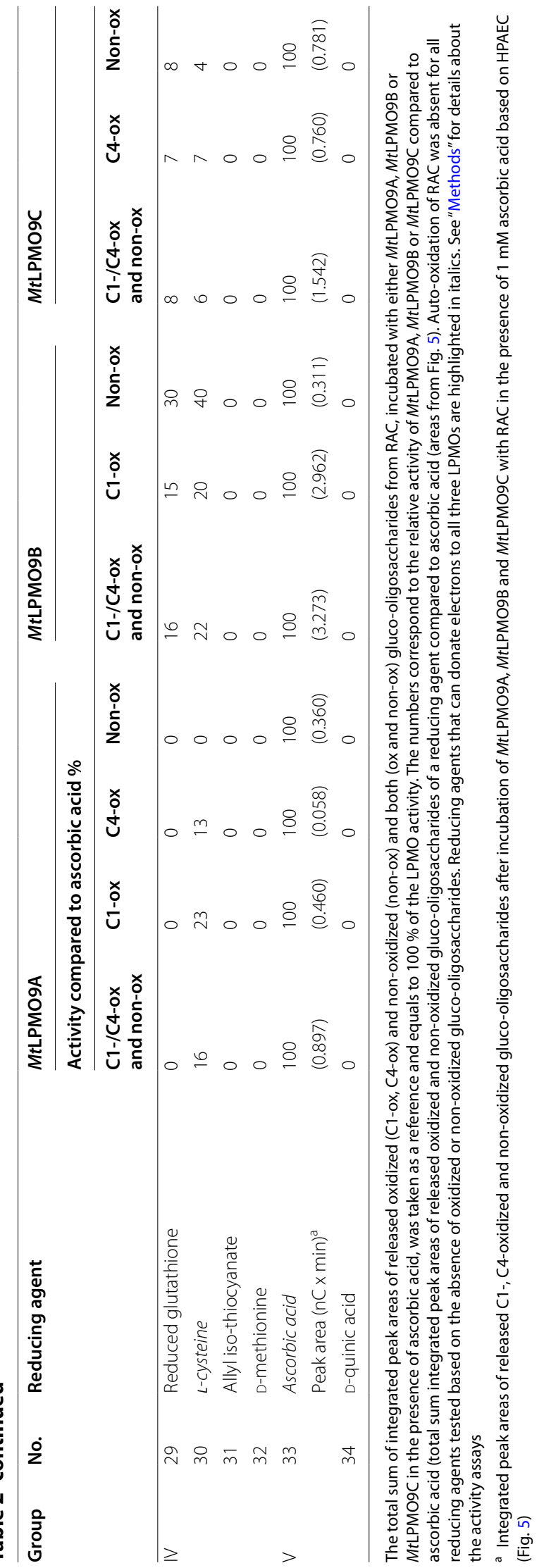




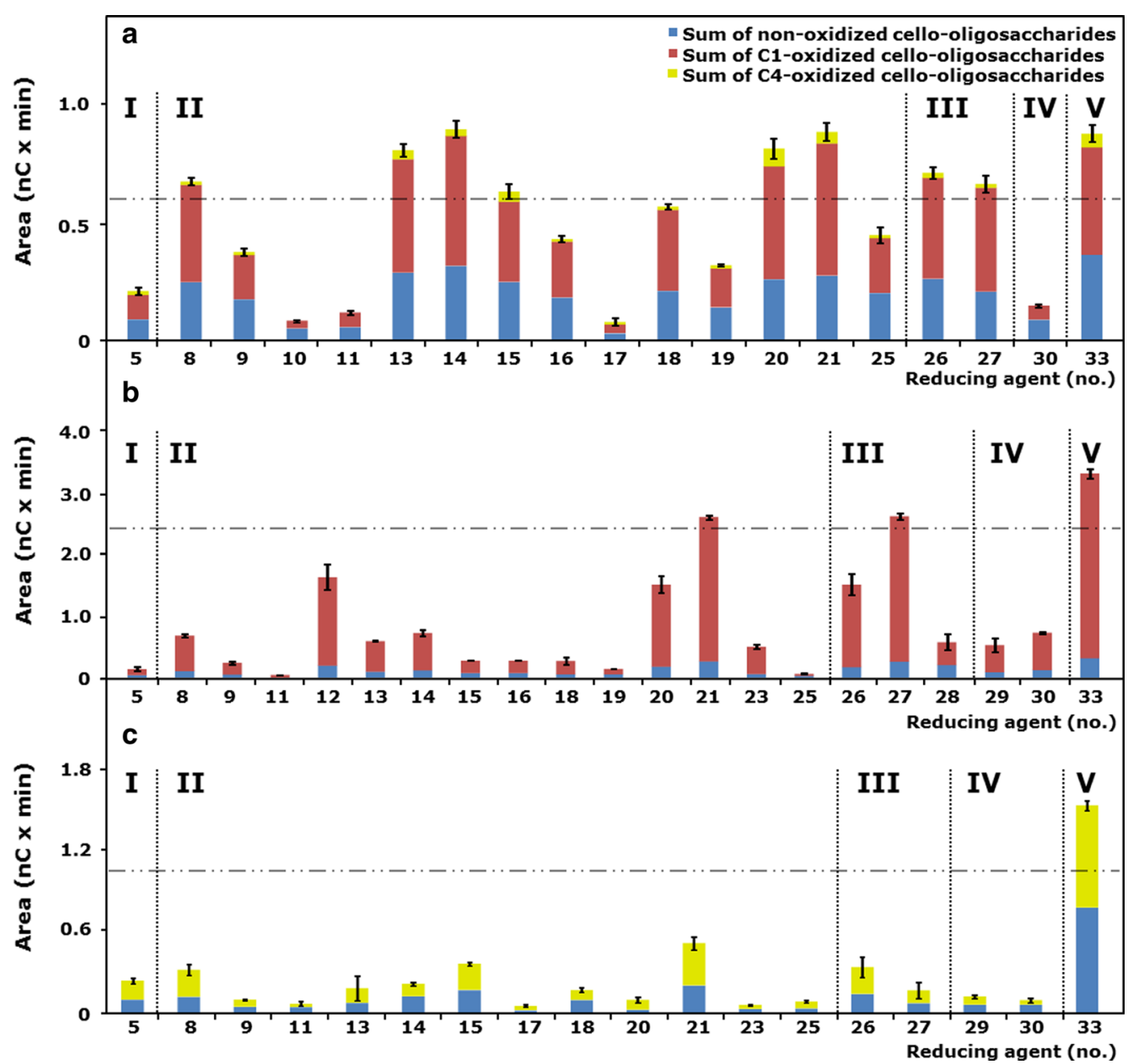

Fig. 5 Released oligosaccharides from RAC incubated with three different MtLPMOs. RAC was incubated with a MtLPMO9A, b MtLPMO9B and c MtLPMO9C using different reducing agents. The total sum is shown of integrated peak areas of released C1- (red), C4- (yellow) oxidized and nonoxidized (blue) gluco-oligosaccharides after incubation of MtLPMO9A, MtLPMO9B and MtLPMO9C $\left(2.5,5\right.$ and $2.5 \mathrm{mg} \times \mathrm{g}^{-1}$ substrate, respectively) with regenerated amorphous cellulose (RAC; $1.5 \mathrm{mg} \times \mathrm{mL}^{-1}$ ) based on HPAEC. The reducing agents (1 mM) are numbered (X-axes) and specified in Table 2. Vertical dotted lines separate reducing agents of the five structural groups (Fig. 4). The threshold (horizontal dashed dotted line) is set to $70 \%$ of the released products from RAC incubated with the MtLPMOs in the presence of ascorbic acid (no. 33) (Table 2). See "Methods" for data analysis. All incubations were performed in duplicates, and the standard deviation is represented by error bars, which correspond to one cumulated SD (error bar $= \pm \mathrm{SDtot} ;$ with SDtot $\left.=\sqrt{ } \mathrm{SD}_{1}^{2}+\mathrm{SD}_{2}^{2}+\ldots\right)$

decomposition of these labile C4-oxidized gluco-oligosaccharides into non-oxidized compounds (Fig. 1) [34].

\section{Substrate specificity and $\mathrm{C} 1-/ \mathrm{C} 4-$-regioselectivity}

$M t \mathrm{LPMO} 9 \mathrm{~A}, M t \mathrm{LPMO}$ B and $M t \mathrm{LPMO} 9 \mathrm{C}$ were compared regarding their substrate specificity and C1-/ C4-regioselectivity of oxidation using a wide range of polysaccharides (Table 1). The activity of MtLPMO9A toward various polysaccharides has already been investigated previously, showing that $M t$ LPMO9A oxidizes cellulose, besides $\beta$ - $(1 \rightarrow 3,1 \rightarrow 4)$-glucan from oat spelt or barley and xyloglucan from tamarind seed, at the $\mathrm{C} 1$ and $\mathrm{C} 4$ position. In addition, $M t$ LPMO9A also oxidized xylan in the presence of cellulose [2]. MtLPMO9B oxidized $\mathrm{RAC}$ at the $\mathrm{C} 1$ position. The presence of the CBM1 linked to $M t$ LPMO9B is not expected to have an impact on the substrate specificity of $M t L P M O 9 B$. It has been shown that the removal of a CBM1 linked to NcLPMO9C, which has a broad substrate specificity, does not affect the ability to oxidize different polysaccharides such as cellulose, cellopentaose or xyloglucan [25]. Besides that, $M t$ LPMO9B shows a typical insertion, which forms the L3 loop region in C4-oxidizing AA9 LPMOs. Compared to the solely C4-oxidizing NcLPMO9C, MtLPMO9B shares within this L3 loop only a low sequence identity $[10,25]$. The L3 loop is known to be an extension 
of the surface-binding site and involved in the binding of soluble polysaccharides such as xyloglucan and cello-oligosaccharides [25-27]. MtLPMO9C also contains the L3 loop and is, like NcLPMO9C, a C4-oxidizing AA9 LPMO (Fig. 2). In addition to cellulose, $M t$ LPMO9C oxidizes to a lower extent hemicellulose like $\beta-(1 \rightarrow 3,1 \rightarrow 4)$-glucan from oat spelt and xyloglucan from tamarind seed at the $\mathrm{C} 4$ position (Additional files 4, 5, 6). The previously published NcLPMO9D (PDB id: 4EIR) from N. crassa shares the highest sequence identity $(83 \%)$ with $M t$ LPMO9C, but unlike $M t$ LPMO9C it has not been tested if NcLPMO9D is active on hemicellulose such as $(1 \rightarrow 3$, $1 \rightarrow 4$ )-glucan $[15,29]$.

\section{Reducing agent specificity}

We show here that three LPMOs from the same organism differ in their reducing agent specificity. Importantly, all three LPMOs are able to utilize various natural phenolic compounds as reducing agents. Most of these reducing agents are present in plants, either free or as lignin building blocks, such as sinapic acid (no. 5), or as flavonoids such as catechin (no. 9) and dopamine (no. 20). This finding is of high relevance as these reducing agents can act as intrinsic electron donors in plant biomass biorefinery [35].

For all three $M t$ LPMOs, phenolic compounds with 1,2-benzenediol and 1,2,3-benzenetriol moieties yielded the highest release of oxidized and non-oxidized glucooligosaccharides from cellulose compared to monophenols or sulfur-containing compounds (Fig. 5; Table 2). This observation may be related to the fact that the donation of an electron by one hydroxyl group leads to a dislocation of the $\pi$-electron sextet, which is energetically unfavorable. It has been shown that monophenols have a higher oxidation potential compared to compounds with 1,2-benzenediol and 1,2,3-benzenetriol moieties [20]. This high oxidation potential of monophenols hinders the reduction of the active site copper of LPMOs. In contrast to monophenols, phenolic compounds with 1,2-benzenediol and 1,2,3-benzenetriol moieties can stabilize the dislocation of $\pi$-electrons by their additional(s) hydroxyl groups due to the resonance effect [36]. Compared to monophenols, these compounds have a low reduction potential and are able to reduce the active site copper of the LPMOs [20]. In addition, the ability of such compounds to donate electrons is also influenced by other electron-donating or electron-withdrawing substituents attached to the aromatic ring $[37,38]$.

Remarkably, the number of reducing agents that gave an activity of $70 \%$ or higher of the LPMO activity obtained with ascorbic acid differed between the three LPMOs (Fig. 5; Table 2). For MtLPMO9A this number was eight (compounds with a benzenediol and benzenetriol moiety), but for $M t \mathrm{LPMO} 9 \mathrm{~B}$ this number was only two (Fig. 5; Table 2). MtLPMO9C oxidizes RAC in the presence of several reducing agents, but, in comparison to $M t \mathrm{LPMO} 9 \mathrm{~A}$ and $M t \mathrm{LPMO} 9 \mathrm{~B}$, none of the reducing agents tested gave an activity of $70 \%$ or higher of the $M t \mathrm{LPMO} 9 \mathrm{C}$-activity obtained with ascorbic acid. Remarkably, we also found reducing agents with a 1,2-benzenediol moiety that are not able to reduce the active site copper of all three $M t L P M O s$ tested (Table 2). These findings cannot be explained by the oxidation potential of the reducing agents alone. Another explanation for the reducing agent preferences among the three LPMOs may result from differences in the protein structure. It has been formerly hypothesized that the binding site of electron-donating proteins, such as CDHs, is located in the surface patch centered around the ProGly-Pro triad, which is highly conserved within the LPMO family [21, 29]. However, recent analysis based on $\mathrm{CDH}$ docking studies and NMR revealed a direct interaction of the $\mathrm{CDH}$ with the LPMO involving a narrow surface patch around the His1, Ala80, His83 and His155 of NcLPMO9C [21, 26]. Indeed, the surface charge distribution obtained from the homology models differs widely among the three $M t L P M O s$, including shape and charge in the vicinity of the above described surface patch (Fig. 6; Additional file 7). MtLPMO9A is strongly positively charged in the vicinity of the copper ion compared to $M t \mathrm{LPMO} 9 \mathrm{~B}$ and $M t \mathrm{LPMO} 9 \mathrm{C}$ (pH 5.0). The charge differences in the vicinity of the copper ion might contribute to the different electron donor specificities of the MtLPMOs.

The incubations performed in this work have been conducted at a single time point $(24 \mathrm{~h})$ and at one $\mathrm{pH}(5.0)$. This $\mathrm{pH}$ plays an important role for the LPMO application, due to the fact that cellulose cleaving cocktails produced by $M$. thermophila $\mathrm{C} 1$ have their $\mathrm{pH}$ optimum around $\mathrm{pH}$ 5.0. It can be expected that this $\mathrm{pH}$ does not represent the optimal condition for each reducing agent tested, since redox potentials of reducing agents are $\mathrm{pH}$ dependent [37]. Furthermore, the product release determined at a single time point $(24 \mathrm{~h})$ does not give information about the progress of the LPMO reaction. The time point of $24 \mathrm{~h}$ could lie in the initial rate period or already at the end point of the LPMO reaction, which highly depends on the reducing agents present during the reaction $[17,33]$. We do not expect a release of non-oxidized or oxidized gluco-oligosaccharides after $24 \mathrm{~h}$ if no products have been released from RAC incubated with LPMOs before that time point (Table 2). Finally, the conditions chosen for all three LPMOs have been the same, which allows the comparison of the LPMO activity in the presence of different reducing agents and the chosen conditions can be considered as industrially relevant. 

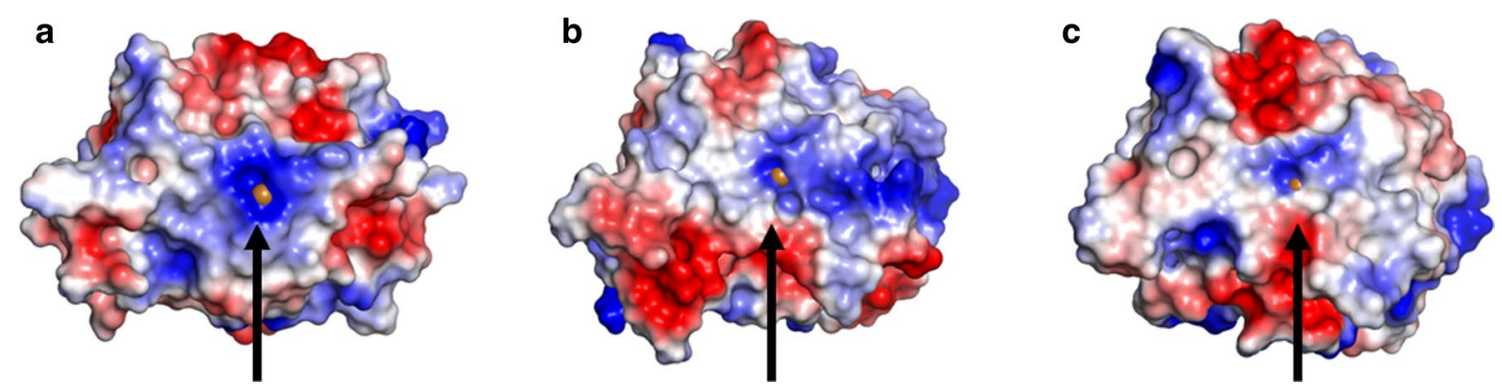

Fig. 6 Cartoons of the surface charge distribution of the structural models of a MtLPMO9A, b MtLPMO9B and c MtLPMO9C. Protein orientation: the flat substrate-binding site (Fig. 3) is located to the front of all three LPMOs and the copper ion is indicated by the black arrow. Recent NMR studies revealed a direct interaction of the reductant CDH with a narrow surface patch in the vicinity of the copper ion [26]. MtLPMO9A is strongly positively charged in the vicinity of the copper ion compared to MtLPMO9B and MtLPMO9C based on the surface charge distribution (pH 5.0). The scaling from the negative and positive electrostatic potential regions are -5 for blue and +5 for the red regions. The electrostatic map was obtained from APBS plugin from PyMOL

\section{Conclusions}

Our findings support the hypothesis that LPMOs do not only vary in their C1-/C4-regioselectivity and substrate specificity, but also in their reducing agent specificity. The mode of action of LPMOs is usually investigated in the presence of ascorbic acid. Here, we found that several reducing agents can donate electrons to the LPMOs with a similar efficiency as the commonly used ascorbic acid. Our findings are of high interest for industrial applications as most of these reducing agents are present in plant biomass and can act as intrinsic mediators in biorefinery processes.

\section{Methods}

\section{Enzyme expression, production and purification}

The purification and activity of MtLPMO9A has been previously described [2]. The homologous expression of $M t$ LPMO9B (UniProt: KX772410) and $M t$ LPMO9C (UniProt: KX772411) was performed using a low protease/low (hemi-) cellulose producing Myceliophthora thermophila $\mathrm{C} 1$ strain, which has been described elsewhere $[39,40]$. The crude enzyme preparations obtained from the fermentation broth were dialyzed against $10 \mathrm{mM}$ potassium phosphate buffer ( $\mathrm{pH}$ 7.0). MtLPMO9B and $M t$ LPMO9C were purified from the corresponding dialyzed enzyme preparations using an ÄKTA-Explorer preparative chromatography system (GE Healthcare, Uppsala, Sweden).

$M t$ LPMO9B was purified in three subsequent chromatographic steps. For the first anion-exchange step, the $M t$ LPMO9B-containing enzyme preparation was loaded on a Source $30 \mathrm{Q}$ column $(50 \mathrm{ml}$, GE Healthcare). A $20 \mathrm{mM}$ potassium phosphate buffer ( $\mathrm{pH} 7.8$ ) was used to pre-equilibrate the column. Elution was performed using a linear gradient from 0 to $1 \mathrm{M} \mathrm{NaCl}$ in $20 \mathrm{mM}$ potassium phosphate buffer $(\mathrm{pH} 7.8)$ at a flow rate of $10 \mathrm{~mL} \times \mathrm{min}^{-1}$ and monitored at 220 and $280 \mathrm{~nm}$. All fractions were collected and immediately stored on ice. Peak fractions were, based on UV $(280 \mathrm{~nm})$, pooled and concentrated by ultrafiltration (Amicon Ultra, molecular mass cut-off of $3 \mathrm{kDa}$, Merck Millipore, Cork, Ireland) at $4{ }^{\circ} \mathrm{C}$. The concentrated pools were analyzed by SDS-PAGE to determine the $M t$ LPMO9B-containing pool (expected molecular mass $30.6 \mathrm{kDa}$ ). After the first purification step, cation exchange chromatography purification was applied. The $M t$ LPMO9B containing pool was subjected to a Source $30 \mathrm{~S}$ column (50 mL, GE Healthcare) for further purification (second step). A $20 \mathrm{mM}$ sodium acetate buffer ( $\mathrm{pH}$ 5.0) was used to pre-equilibrate the column. The elution was performed by using a linear gradient from 0 to $1 \mathrm{M} \mathrm{NaCl}$ in $20 \mathrm{mM}$ sodium acetate (pH 5.0) at a flow rate of $5 \mathrm{~mL} \times \mathrm{min}^{-1}$. Elution was monitored at 220 and $280 \mathrm{~nm}$. Fractions obtained $(10 \mathrm{~mL})$ were immediately stored on ice. Peak fractions were pooled, concentrated and analyzed by SDS-PAGE as described above. In a third purification step, the MtLPMO9Bcontaining fraction was bound to a Source $30 \mathrm{~S}$ column (50 mL, GE Healthcare) using a $10 \mathrm{mM}$ sodium acetate buffer ( $\mathrm{pH}$ 5.0) after pre-equilibration. After protein application, the column was washed with 20 column volumes of starting buffer. Elution was performed using a linear gradient from 0 to $1 \mathrm{M} \mathrm{KCl}$ in $20 \mathrm{mM}$ sodium acetate $(\mathrm{pH} 5.0)$ at a flow rate of $5 \mathrm{~mL} \times \mathrm{min}^{-1}$ and monitored at 220 and $280 \mathrm{~nm}$. Fractions $(3 \mathrm{~mL})$ were immediately stored on ice. Peak fractions were pooled, concentrated and analyzed by SDS-PAGE as described above. 
$M t$ LPMO9C was purified in four subsequent chromatographic steps. As a first size exclusion chromatography (SEC) purification step, the $M t$ LPMO9C-rich enzyme preparation $\left(40 \mathrm{mg} \times \mathrm{mL}^{-1}\right)$ was loaded onto a selfpacked Superdex TM-75 column $(100 \times 3 \mathrm{~cm}$ internal diameter, GE Healthcare) and eluted at $5 \mathrm{~mL} \times \mathrm{min}^{-1}$ with a $10 \mathrm{mM}$ potassium phosphate buffer $(\mathrm{pH} 7.0)$. Fractions $(5 \mathrm{~mL})$ were immediately stored on ice. Peak fractions were, based on UV (280 nm), pooled and concentrated by ultrafiltration as described above. The concentrated pools were analyzed by SDS-PAGE to determine the $M t$ LPMO9C-containing pool (expected molecular mass $23.5 \mathrm{kDa}$ ). For the second SEC purification step, the $M t$ LPMO9C-containing pool was loaded again on the Superdex TM-75 column under the same conditions. Fractions $(5 \mathrm{~mL})$ were immediately stored on ice. Peak fractions were pooled, concentrated and analyzed by SDS-PAGE to determine the $M t$ LPMO9C-containing pool as described above. The $M t$ LPMO9C-containing pool was dialyzed against a $20 \mathrm{mM}$ Tris- $\mathrm{HCl}$ buffer $(\mathrm{pH}$ 8.4) using ultrafiltration (Amicon Ultra, molecular mass cutoff of $3 \mathrm{kDa}$ ). The dialyzed $M t \mathrm{LPMO} 9 \mathrm{C}$ fraction was, for the third purification step, subjected to a Resource Q column $(30 \times 16 \mathrm{~mm}$ internal diameter, GE Healthcare), which was pre-equilibrated in $20 \mathrm{mM}$ Tris- $\mathrm{HCl}$ buffer ( $\mathrm{pH}$ 8.4) (third step). Elution was performed with a linear gradient from 0 to $1 \mathrm{M} \mathrm{NaCl}$ in $20 \mathrm{mM}$ Tris- $\mathrm{HCl}(\mathrm{pH}$ 8.4) over 20 column volumes at $6 \mathrm{~mL} \times \mathrm{min}^{-1}$. Fractions $(3 \mathrm{~mL})$ were immediately stored on ice. Peak fractions were pooled, concentrated and analyzed by SDS-PAGE to determine the $M t \mathrm{LPMO} 9 \mathrm{C}$-containing pool as described above. The $M t$ LPMO9C-containing fraction was dialyzed against a $20 \mathrm{mM}$ potassium phosphate buffer $(\mathrm{pH}$ 7.0) using ultrafiltration (Amicon Ultra, molecular mass cut-off of $3 \mathrm{kDa}$ ) and subjected to a Resource Q column $(30 \times 16 \mathrm{~mm}$ internal diameter, GE Healthcare). The column was equilibrated using a $20 \mathrm{mM}$ potassium phosphate buffer ( $\mathrm{pH}$ 7.0) and elution was performed using a linear gradient over 20 column volumes at $6 \mathrm{~mL} \times \mathrm{min}^{-1}$. Fractions $(3 \mathrm{~mL})$ were immediately stored on ice. Peak fractions were pooled, concentrated and analyzed by SDS-PAGE as described above.

\section{Protein analysis}

The protein content of $M t \mathrm{LPMO} 9 \mathrm{~B}$ and $M t \mathrm{LPMO} C \mathrm{C}$ was determined as described previously using a BCA Protein Assay Kit [2]. Furthermore, the purity of the enzymes was analyzed by sodium dodecyl sulfate polyacrylamide gel electrophoresis (SDS-PAGE) as described before [2]. Pure $M t \mathrm{LPMO} 9 \mathrm{~B}$ and $M t \mathrm{LPMO} C \mathrm{C}$ fractions were analyzed by LC-mass spectrometry confirming the presence of the two LPMOs by 'The Scripps Research Institute' (San Diego, CA, USA).

\section{LC/ESI-MS}

Purified $M t$ LPMO9B and $M t$ LPMO9C preparations $\left(2.5 \mathrm{mg} \mathrm{mL}^{-1}\right.$ in $0.1 \%(\mathrm{v} / \mathrm{v})$ trifluoroacetic acid) were analyzed using a liquid chromatography/electron spray ionization-mass spectrometry (LC/ESI-MS) as described previously [2].

\section{Reducing agents}

Reducing agents were supplied by Sigma-Aldrich (Steinheim, Germany), unless stated otherwise. Taxifolin was purchased from Extrasynthese (Genay, France), catechol hydrate and chlorogenic acid from Thermo Fisher Scientific (Waltham, MA USA), tannic acid from BDH Chemical Ltd. (Poole, England) and naringin from Fluka Chemie (Buches, Switzerland).

\section{Carbohydrates}

OSX, BiWX, Avicel PH-101, xylo-oligosaccharides (DP1$5)$ and $\beta-(1 \rightarrow 4)$-linked gluco-oligosaccharides (DP1-5) were supplied by Sigma-Aldrich. WAX and $\beta-(1 \rightarrow 3$, $1 \rightarrow 4$ )-glucan from barley and oat spelt (both medium viscosity) were purchased from Megazyme (Bray, Ireland). Xyloglucan from tamarind seed was obtained from Dainippon Sumitomo Pharma (Osaka, Japan). Regenerated amorphous cellulose (RAC) was prepared from Avicel PH-101 as described [2, 41]. Gluconic acid was purchased from Sigma-Aldrich and cellobionic acid ammonium salt from Toronto Research Chemicals (Toronto, Ontario, Canada).

\section{MtLPMO9A, MtLPMO9B and MtLPMO9C activity assays}

Substrates (see figure captions) were dissolved in $50 \mathrm{mM}$ ammonium acetate buffer ( $\mathrm{pH}$ 5.0) to a concentration of 1-2 mg $\times \mathrm{mL}^{-1}$, with or without addition of reducing agents listed in Table 1 (final concentration of $1 \mathrm{mM}$ ). $M t$ LPMO9A, $M t$ LPMO9B or $M t$ LPMO9C was added (2.5-10.0 $\mu$ g of protein $\mathrm{mg}^{-1}$ substrate, for details see figure captions) and incubated for $24 \mathrm{~h}$ at $50{ }^{\circ} \mathrm{C}$ in a headover-tail Stuart rotator in portions of $1 \mathrm{~mL}$ total volume (Bibby Scientific, Stone, UK) at $20 \mathrm{rpm}$. Supernatants of all incubations with and without reducing agent in the presence of LPMOs and of substrates incubated with and without reducing agents in the absence of LPMOs were analyzed by HPAEC and MALDI-TOF MS. The enzyme reactions were stopped by storing samples at $-24{ }^{\circ} \mathrm{C}$. All following sample treatments were performed on ice.

\section{Structural modeling}

The structural model of $M t$ LPMO9B was made using the available structure of NcLPMO9C from Neurospora crassa [25] (Protein Data Bank entry: 4D7U) as a template, which scored highest in the BLAST search of $M t$ LPMO9B against the Protein Data Bank (41 \% amino 
acid identity). $M t \mathrm{LPMO}$ C was, like $M t \mathrm{LPMO} 9 \mathrm{~B}$, generated based on NcLPMO9C [25] (PDB entry: 4D7U, $46 \%$ amino acid identity). All models were created using Modeller version 9.14 [42]. Multiple comparative models were generated, after which the model with the lowest corresponding DOPE score [43] was selected for image generation using Pymol (Pymol, The PyMOL Molecular Graphics System, Version 1.5.0.4 Schrödinger, New York, NY, USA). The following settings were applied to model the surface charge distribution of the LPMOs: The protonation states of the titratable groups at $\mathrm{pH} 5.0$ of $M t \mathrm{LPMO} 9 \mathrm{~A}, M t \mathrm{LPMO} 9 \mathrm{~B}$ and $M t \mathrm{LPMO}$ C, respectively, were calculated using $\mathrm{H}^{++}$-server with default settings [44-47]. The server pdb-ouput files were used to generate a surface image colored by charge (range between -5 and 5) using the Pymol APBS-tool (Version 1.4r1 L, Schrödinger).

\section{Hpaec}

Enzyme digests were analyzed by high-performance anion-exchange chromatography (HPAEC) with pulsed amperometric detection (PAD) using an HPAEC system (ICS-5000, Dionex, Sunnyvale, CA, USA) as described previously [2]. The temperature of the autosampler was set to $6{ }^{\circ} \mathrm{C}$. For the analysis of C4-oxidized glucooligosaccharides released by $M t \mathrm{LPMO}$ C, a longer gradient was used. The gradient elution program was as follows: 0-45 min, linear gradient $0-250 \mathrm{mM} \mathrm{NaOAc}$; $45-52$ min isocratic gradient $400-1000 \mathrm{mM} \mathrm{NaOAc}$. This was followed by equilibration $(13 \mathrm{~min})$ of the column with the starting conditions. The assignment of the $\mathrm{C} 1$ - and C4-oxidized gluco-oligosaccharides using HPAEC is based on previous publications $[2,8,15$, 17], while gluconic and cellobionic acids were assigned by available standards (see "Methods"). All incubations were performed in duplicate. Areas were analyzed to determine the effect of the reducing agents on the release of oxidized and non-oxidized gluco-oligosaccharides from RAC incubated with MtLPMO9A, $M t \mathrm{LPMO} 9 \mathrm{~B}$ or $M t \mathrm{LPMO}$ C. Standard deviations are represented (Fig. 5) by error bars, which correspond to one cumulated SD (error bar $= \pm$ SDtot; with SDtot $=\sqrt{ } \mathrm{SD} 12+\mathrm{SD} 22+\ldots)$.

\section{MALDI-TOF MS}

The analysis of substrates incubated with either MtLPMO9B or $M t$ LPMO9C was performed using matrixassisted laser desorption ionization-time of flight mass spectrometry (MALDI-TOF MS, Bruker Daltonics) as described previously [2]. Masses of lithium-adducted C1or C4-oxidized gluco-oligosaccharides for RAC incubated with $M t \mathrm{LPMO9B}$ or $M t \mathrm{LPMO}$ C, respectively, were determined and assigned as described previously [2].

\section{Additional files}

Additional file 1: Figure S1. SDS-PAGE of MtLPMO9B and MtLPMO9C fractions during enzyme purification. MtLPMO9B and MtLPMO9C were purified by multiple chromatographic steps from the crude enzyme extract of MtLPMO9B (lane 1) and MtLPMO9C (lane 2). The pools of MtLPMO9B (lane 4) or MtLPMO9C (lane 5), used for various experiments, showed a single protein band with apparent molecular masses of 32 and $25 \mathrm{kDa}$, respectively (black arrows). The Precision Plus Protein (Bio-Rad Laboratories) was used as a marker. (lane 3 and 6). For more details about protein purification see Methods.

Additional file 2: Figure S2. LC/ESI-MS analysis of MtLPMO9B and MtLPMO9C. The purified a MtLPMO9B- and b MtLPMO9C-preparation was analyzed by LC/UV/ESI-MS using an AQUITY UPLC separation system and a SYNAPT ion mobilty mass spectrometer. The weighted average mass of MtLPMO9B and MtLPMO9C were 32,765 Da and 24,640 Da, respectively. ESI MS spectras ( $m / z$ values) of MtLPMO9B and MtLPMO9C show the presence of multiple glycations (+162 Da, hexose (180 Da) - water (18 Da)) of both LPMOs. Up to 13 and 5 glycosyl units are attached to MtLPMO9B and MtLPMO9C, respectively.

Additional file 3: Figure S3. MALDI-TOF mass spectrum of RAC incubated with MtLPMO9B and MtLPMO9C in the presence of ascorbic acid. a MtLPMO9B incubated with RAC (RAC; $2 \mathrm{mg} \mathrm{x} \mathrm{g}^{-1}$ ) in the presence of ascorbic acid. Clusters of C1-oxidized (GlcOS ${ }_{n}^{\#}$ ) and non-oxidized (GlcOS $n$ ) gluco-oligosaccharides were determined as their lithium (Li) adducts. Double Li-adducts are formed by exchanging a $\mathrm{H}^{+}$ion for another $\mathrm{Li}^{+}$ ion (marked as GlcOS $\#$ ). b MtLPMO9C incubated with RAC (RAC; 2 mg x $\mathrm{g}^{-1}$ ) in the presence of ascorbic acid. Clusters of gluco-oligosaccharides oxidized at the $\mathrm{C} 4$ position $\left(\mathrm{GlCOS}_{n}{ }^{*}\right.$ ) and non-oxidized gluco-oligosaccharides $\left(\mathrm{GlCOS}_{n}\right)$ were determined as their lithium adducts. $a$ and $b$

Clusters of non-oxidized and oxidized gluco-oligosaccharides differ by a mass difference of one glucose unit (GlcOS $1,180 \mathrm{Da}-16 \mathrm{Da}=162 \mathrm{Da})$. See Fig. 1 for more details.

Additional file 4: Figure S4. HPAEC elution patterns of $\beta-(1 \rightarrow 3$, $1 \rightarrow 4$ )-glucan from oat spelt and xyloglucan incubated with MtLPMO9C. Incubation of a oat spelt $\beta$ - $(1 \rightarrow 3,1 \rightarrow 4)$-glucan $\left(2 \mathrm{mg} \times \mathrm{mL}^{-1}\right)$ and b xyloglucan from tamarind seed (XG; $2 \mathrm{mg} \times \mathrm{mL}^{-1}$ ) with MtLPMO9C (10 $\mathrm{mg} \times \mathrm{g}^{-1}$ substrate) with ascorbic acid (1 mM) or without. Samples were incubated in a $50 \mathrm{mM}$ ammonium acetate (pH 5.0) for $24 \mathrm{~h}$ at $52{ }^{\circ} \mathrm{C}$. a Numerous products (black arrows) were formed from oat spelt $\beta$ - $(1 \rightarrow 3,1 \rightarrow 4)$-glucan incubated with MtLPMO9C in the presence of ascorbic acid compared to oat spelt $\beta$-( $1 \rightarrow 3,1 \rightarrow 4)$-glucan without MtLPMO9C addition in the presence of ascorbic acid. No oligosaccharides were released if oat spelt $\beta$-( $1 \rightarrow 3,1 \rightarrow 4)$-glucan was incubated with MtLPMO9C in the absence of ascorbic acid. $b$ Incubation of XG with MtLP$\mathrm{MO} C \mathrm{C}$ in the presence of ascorbic acid released numerous products (black arrows) which were not present if XG was incubated with MtLPMO9C in the absence of ascorbic acid. No oligosaccharides were formed from XG incubated with MtLPMO9C in the absence of ascorbic acid.

Additional file 5: Figure S5. MALDI-TOF mass spectrum of $\beta-(1 \rightarrow 3$, $1 \rightarrow 4$ )-glucan from oat spelt incubated with MtLPMO9C. a MtLPMO9B incubated with oat spelt $\beta-(1 \rightarrow 3,1 \rightarrow 4)$-glucan $\left(2 \mathrm{mg} \mathrm{x} \mathrm{mL}^{-1}\right)$ in the presence of ascorbic acid. Clusters of C4-oxidized (GlcOS*) and nonoxidized $\left(\mathrm{GlCOS}_{n}\right)$ gluco-oligosaccharides were determined as their lithium (Li) adducts. Clusters of non-oxidized and C4-oxidized glucooligosaccharides differ by a mass difference of one glucose unit (GlcOS, $180 \mathrm{Da}-16 \mathrm{Da}=162 \mathrm{Da}$ ). b (enlargement of a) Several additional peaks were determined showing the characteristic 2 Da lower mass as reported for C4-oxidized products $\left(\mathrm{GlCOS}_{n}{ }^{*}=\mathrm{GlCOS}_{n}-2 \mathrm{Da}\right)$. See Methods for more details.

Additional file 6: Figure S6. MALDI-TOF mass spectrum of xyloglucan incubated with MtLPMO9C. a MtLPMO9C incubated with xyloglucan

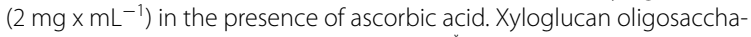
ride (XG-OS) clusters of C4-oxidized (XG-OS*) and non-oxidized (XG-OS ${ }_{n}$ ) oligosaccharides were determined ( $\mathrm{m} / \mathrm{z}$ values) as their lithium (Li) adducts. b (enlargement of a) Several additional peaks were annotated showing the characteristic 2 Da lower mass as reported for C4-oxidized 
products $\left(\mathrm{GlCOS}_{n}{ }^{*}=\mathrm{GlCOS}_{n}-2 \mathrm{Da}\right)$. An identification of non-oxidized and C4-oxidized gluco-oligosaccharides of different substituted xyloglucan oligosaccharides based on Fry et al. [49] remains limited due to the low amounts of products released from xyloglucan incubated with MtLPMO9C (Additional file 4) and the therefore impossible $\mathrm{MS}^{2}$-fragmentation [49]. Compounds annotated as $\mathrm{GIOS}_{n} \mathrm{XOS}_{n}$ indicate the number of expected hexoses and pentoses to be present in xyloglucan oligosaccharides. Samples were incubated in $50 \mathrm{mM}$ ammonium acetate buffer ( $\mathrm{pH}$ 5.0) containing $1 \mathrm{mM}$ ascorbic acid for $24 \mathrm{~h}$ at $52^{\circ} \mathrm{C}$. See Methods for more details.

Additional file 7: Figure S7. Cartoons of the highly conserved surface patch near the Gly-Pro-Gly triad. Surface charge distribution and enlargement (in brackets) of a MtLPMO9A (d), b MtLPMO9B (e) and c MtLPMO9C (f) emphasize the highly conserved surface patch near the Gly-Pro-Gly triad, positioned in the amino acid sequence around residue number 200 $[21,29]$. The scaling from the negative and positive electrostatic potential regions are -5 for blue and +5 for the red regions. The electrostatic map was obtained from APBS plugin from PyMOL. Protein orientation: the flat substrate-binding site is located at the bottom of all three LPMOs indicated by the black arrow.

\section{Abbreviations}

AA: auxiliary activity; AEC: anion-exchange chromatography; AUC: area under the curve; BiWX: birchwood glucuronoxylan; CAZy: carbohydrate-active enzymes; CBM: carbohydrate-binding module; $\mathrm{CDH}$ : cellobiose dehydrogenase; GH: glycoside hydrolases; GlcAmeXOS $:$ 4-O-methylglucoronic acid containing xylo-oligosaccharides; GlcOS : gluco-oligosaccharides; \#: C1

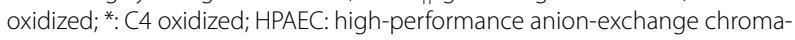
tography; LC/ESI-MS: liquid chromatography/electrospray ionization-mass spectrometry; LPMO: lytic polysaccharide monooxygenase; MALDI-TOF MS: matrix-assisted laser desorption ionization-time of flight mass spectrometry; OSX: oat spelt xylan; PAD: pulsed amperometric detection; PMO: polysaccharide monooxygenase; RAC: regenerated amorphous cellulose; SEC: size exclusion chromatography; SDS-PAGE: sodium dodecyl sulfate-polyacrylamide gel electrophoresis; WAX: wheat arabinoxylan.

\section{Authors' contributions}

All authors contributed to this study. MF, MJK, AHW, JV, SWAH, JPV, WJHB, HG and MAK made substantial contribution to the conception and design. MF carried out the experiments, performed the data analysis and prepared the manuscript. MF and AHW prepared the structural models and surface charge distribution cartoons. All authors were involved in drafting the manuscript or revising it critically for the intellectual content. All authors gave their approval to the final version. All authors read and approved the final manuscript.

\section{Author details}

${ }^{1}$ Laboratory of Food Chemistry, Wageningen University, Bornse Weilanden 9, 6708 WG Wageningen, The Netherlands. ${ }^{2}$ DuPont Industrial Biosciences, Nieuwe Kanaal 7-S, 6709 PA Wageningen, The Netherlands. ${ }^{3}$ Laboratory of Biochemistry, Wageningen University, Stippeneng 4, 6708 WE Wageningen, The Netherlands. ${ }^{4}$ Fungal Genetics \& Technology Consultancy, P.O. Box 39b, 6700 AJ Wageningen, The Netherlands.

\section{Acknowledgements}

This research was supported by a grant from the VLAG Graduate School (Advanced Studies in Food Technology, Agrobiotechnology, Nutrition and Health Sciences) of Wageningen University (Wageningen, The Netherlands).

\section{Availability of supporting data}

Supporting data will be provided as Additional files 1-7.

\section{Competing interests}

The authors declare that they have no competing interests.

Received: 10 June 2016 Accepted: 19 August 2016

Published online: 31 August 2016

\section{References}

1. Hinz SWA, Pouvreau L, Joosten R, Bartels J, Jonathan MC, Wery J, et al. Hemicellulase production in Chrysosporium lucknowense C1. J Cereal Sci. 2009;50(3):318-23. doi:10.1016/j.jcs.2009.07.005.

2. Frommhagen M, Sforza S, Westphal AH, Visser J, Hinz SW, Koetsier MJ, et al. Discovery of the combined oxidative cleavage of plant xylan and cellulose by a new fungal polysaccharide monooxygenase. Biotechnol Biofuels. 2015;8:101. doi:10.1186/s13068-015-0284-1.

3. Berka RM, Grigoriev IV, Otillar R, Salamov A, Grimwood J, Reid I, et al. Comparative genomic analysis of the thermophilic biomass-degrading fungi Myceliophthora thermophila and Thielavia terrestris. Nat Biotechnol. 2011;29(10):922-7. doi:10.1038/nbt.1976.

4. Parthasarathi R, Bellesia G, Chundawat SPS, Dale BE, Langan P, Gnanakaran S. Insights into hydrogen bonding and stacking interactions in cellulose. J Phys Chem A. 2011;115(49):14191-202. doi:10.1021/jp203620x.

5. CAZy. Glycoside hydrolase family classification. 2014. http://www.cazy. org/Glycoside-Hydrolases.html. Accessed 17 Jul 2016.

6. Vaaje-Kolstad G, Westereng B, Horn SJ, Liu Z, Zhai H, Sorlie M, et al. An oxidative enzyme boosting the enzymatic conversion of recalcitrant polysaccharides. Science. 2010;330(6001):219-22. doi:10.1126/ science.1192231.

7. Westereng B, Ishida T, Vaaje-Kolstad G, Wu M, Eijsink VG, Igarashi K, et al. The putative endoglucanase PCGH61D from Phanerochaete chrysosporium is a metal-dependent oxidative enzyme that cleaves cellulose. PLOS ONE. 2011;6(11):e27807. doi:10.1371/journal.pone.0027807.

8. Vu W, Beeson WT, Phillips CM, Cate JH, Marletta MA. Determinants of regioselective hydroxylation in the fungal polysaccharide monooxygenases. J Am Chem Soc. 2014;136(2):562-5. doi:10.1021/ja409384b.

9. Isaksen T, Westereng B, Aachmann FL, Agger JW, Kracher D, Kittl R, et al. A C4-oxidizing lytic polysaccharide monooxygenase cleaving both cellulose and cello-oligosaccharides. J Biol Chem. 2014;289(5):2632-42. doi:10.1074/jbc.M113.530196.

10. Agger JW, Isaksen T, Varnai A, Vidal-Melgosa S, Willats WG, Ludwig R, et al. Discovery of LPMO activity on hemicelluloses shows the importance of oxidative processes in plant cell wall degradation. Proc Natl Acad Sci USA. 2014;111(17):6287-92. doi:10.1073/pnas.1323629111.

11. Forsberg Z, Rohr AK, Mekasha S, Andersson KK, Eijsink VG, Vaaje-Kolstad $\mathrm{G}$, et al. Comparative study of two chitin-active and two celluloseactive AA10-type lytic polysaccharide monooxygenases. Biochemistry. 2014;53(10):1647-56. doi:10.1021/bi5000433.

12. Vu W, Beeson WT, Span EA, Farquhar ER, Marletta MA. A family of starch-active polysaccharide monooxygenases. Proc Natl Acad Sci USA. 2014;111(38):13822-7. doi:10.1073/pnas.1408090111.

13. Forsberg Z, Vaaje-Kolstad G, Westereng B, Bunaes AC, Stenstrom Y, MacKenzie A, et al. Cleavage of cellulose by a CBM33 protein. Protein Sci. 2011;20(9):1479-83. doi:10.1002/pro.689.

14. Quinlan RJ, Sweeney MD, Lo Leggio L, Otten H, Poulsen JC, Johansen $\mathrm{KS}$, et al. Insights into the oxidative degradation of cellulose by a copper metalloenzyme that exploits biomass components. Proc Natl Acad Sci USA. 2011;108(37):15079-84. doi:10.1073/pnas.1105776108.

15. Phillips CM, Beeson WT, Cate JH, Marletta MA. Cellobiose dehydrogenase and a copper-dependent polysaccharide monooxygenase potentiate cellulose degradation by Neurospora crassa. ACS Chem Biol. 2011;6(12):1399-406. doi:10.1021/cb200351y.

16. Beeson WT, Phillips CM, Cate JH, Marletta MA. Oxidative cleavage of cellulose by fungal copper-dependent polysaccharide monooxygenases. J Am Chem Soc. 2012;134(2):890-2. doi:10.1021/ja210657t.

17. Bennati-Granier C, Garajova S, Champion C, Grisel S, Haon M, Zhou S, et al. Substrate specificity and regioselectivity of fungal AA9 lytic polysaccharide monooxygenases secreted by Podospora anserina. Biotechnol Biofuels. 2015;8:90. doi:10.1186/s13068-015-0274-3.

18. Rodriguez-Zuniga UF, Cannella D, Giordano RdC, Giordano RdLC, Jorgensen $H$, Felby C. Lignocellulose pretreatment technologies affect the level of enzymatic cellulose oxidation by LPMO. Green Chem. 2015;17(5):2896-903. doi:10.1039/c4gc02179g.

19. Westereng B, Cannella D, Wittrup Agger J, Jørgensen H, Larsen Andersen $M$, Eijsink VGH, et al. Enzymatic cellulose oxidation is linked to lignin by long-range electron transfer. Sci Rep. 2015;5:18561. doi:10.1038/ srep18561. 
20. Kracher D, Scheiblbrandner S, Felice AKG, Breslmayr E, Preims M, Ludwicka $\mathrm{K}$, et al. Extracellular electron transfer systems fuel cellulose oxidative degradation. Science. 2016. doi:10.1126/science.aaf3165.

21. Tan TC, Kracher D, Gandini R, Sygmund C, Kittl R, Haltrich D, et al. Structural basis for cellobiose dehydrogenase action during oxidative cellulose degradation. Nat Commun. 2015. doi:10.1038/ncomms8542.

22. Langston JA, Shaghasi T, Abbate E, Xu F, Vlasenko E, Sweeney MD. Oxidoreductive cellulose depolymerization by the enzymes cellobiose dehydrogenase and glycoside hydrolase 61. Appl Environ Microbiol. 2011;77(19):7007-15. doi:10.1128/AEM.05815-11.

23. Cannella D, Mollers KB, Frigaard NU, Jensen PE, Bjerrum MJ, Johansen KS, et al. Light-driven oxidation of polysaccharides by photosynthetic pigments and a metalloenzyme. Nat Commun. 2016. doi:10.1038/ ncomms11134.

24. Wu M, Beckham GT, Larsson AM, Ishida T, Kim S, Payne CM, et al. Crystal structure and computational characterization of the lytic polysaccharide monooxygenase GH61D from the Basidiomycota fungus Phanerochaete chrysosporium. J Biol Chem. 2013;288(18):12828-39. doi:10.1074/jbc. M113.459396.

25. Borisova AS, Isaksen T, Dimarogona M, Kognole AA, Mathiesen G, Varnai A, et al. Structural and functional characterization of a lytic polysaccharide monooxygenase with broad substrate specificity. J Biol Chem. 2015. doi:10.1074/jbc.M115.660183.

26. Courtade G, Wimmer R, Røhr ÅK, Preims M, Felice AKG, Dimarogona M, et al. Interactions of a fungal lytic polysaccharide monooxygenase with $\beta$-glucan substrates and cellobiose dehydrogenase. Proc Natl Acad Sci. 2016;113(21):5922-7. doi:10.1073/pnas.1602566113.

27. Frandsen KEH, Simmons TJ, Dupree P, Poulsen J-CN, Hemsworth GR, Ciano $L$, et al. The molecular basis of polysaccharide cleavage by lytic polysaccharide monooxygenases. Nat Chem Biol. 2016;12:298-303. doi:10.1038/nchembio.2029.

28. Hemsworth GR, Davies GJ, Walton PH. Recent insights into coppercontaining lytic polysaccharide mono-oxygenases. Curr Opin Struct Biol. 2013;23(5):660-8. doi:10.1016/j.sbi.2013.05.006.

29. Li X, Beeson WT IV, Phillips CM, Marletta MA, Cate JHD. Structural basis for substrate targeting and catalysis by fungal polysaccharide monooxygenases. Structure. 2012;20(6):1051-61. doi:10.1016/j.str.2012.04.002.

30. Lo Leggio L, Simmons TJ, Poulsen JCN, Frandsen KEH, Hemsworth GR, Stringer MA, et al. Structure and boosting activity of a starch-degrading lytic polysaccharide monooxygenase. Nat Commun. 2015;6:5961. doi:10.1038/ncomms6961.

31. Couturier M, Feliu J, Haon M, Navarro D, Lesage-Meessen L, Coutinho PM, et al. A thermostable GH45 endoglucanase from yeast: impact of its atypical multimodularity on activity. Microb Cell Fact. 2011;10(1):1-12. doi:10.1186/1475-2859-10-103.

32. Taylor CB, Talib MF, McCabe C, Bu L, Adney WS, Himmel ME, et al. Computational investigation of glycosylation effects on a family 1 carbohydratebinding module. J Biol Chem. 2012;287(5):3147-55. doi:10.1074/jbc. M111.270389.

33. Crouch LI, Labourel A, Walton PH, Davies GJ, Gilbert HJ. The contribution of non-catalytic carbohydrate binding modules to the activity lytic polysaccharide monooxygenases. J Biol Chem. 2016. doi:10.1074/jbc. M115.702365.
34. Westereng B, Arntzen MØ, Aachmann FL, Várnai A, Eijsink VGH, Agger JW. Simultaneous analysis of C1 and C4 oxidized oligosaccharides, the products of lytic polysaccharide monooxygenases acting on cellulose. J Chromatogr A. 2016. doi:10.1016/j.chroma.2016.03.064.

35. Wang W, Yang S, Hunsinger GB, Pienkos PT, Johnson DK. Connecting lignin-degradation pathway with pretreatment inhibitor sensitivity of Cupriavidus necator. Front Microbiol. 2014. doi:10.3389/fmicb.2014.00247.

36. Ingold CK. Principles of an electronic theory of organic reactions. Chem Rev. 1934;15(2):225-74. doi:10.1021/cr60051a003.

37. Steenken S, Neta P. One-electron redox potentials of phenols. Hydroxyand aminophenols and related compounds of biological interest. J Physic Chem. 1982;86(18):3661-7. doi:10.1021/j100215a033.

38. Hansch C, Leo A, Taft RW. A survey of Hammett substituent constants and resonance and field parameters. Chem Rev. 1991;91(2):165-95. doi:10.1021/cr00002a004.

39. Visser H, Joosten V, Punt PJ, Gusakov AV, Olson PT, Joosten R, et al. Development of a mature fungal technology and production platform for industrial enzymes based on a Myceliophthora thermophila isolate, previously known as Chrysosporium lucknowense C1. Indus Biotechnol. 2011;7:214-23. doi:10.1089/ind.2011.0003.

40. Punt PJ, Burlingame RP, Pynnonen CM, Olson PT, Wery J, Visser J, Heinrich et al. Chrysosporium lucknowense protein production system. 2010. Patent WO/2010/107303.

41. Zhang YHP, Cui J, Lynd LR, Kuang LR. A transition from cellulose swelling to cellulose dissolution by o-phosphoric acid: evidence from enzymatic hydrolysis and supramolecular structure. Biomacromolecules. 2006;7(2):644-8. doi:10.1021/bm050799c.

42. Sali A. Comparative protein modeling by satisfaction of spatial restraints. Mol Med Today. 1995;1(6):270-7. doi:10.1016/S1357-4310(95)91170-7.

43. Eswar N, Webb B, Marti-Renom MA, Madhusudhan MS, Eramian D, Shen MY, et al. Comparative protein structure modeling using MODELLER. Curr Protoc Bioinformatics. 2007. doi:10.1002/0471140864.ps0209s50.

44. Gordon JC, Myers JB, Folta T, Shoja V, Heath LS, Onufriev A. H++: a server for estimating $\mathrm{pK}(\mathrm{a}) \mathrm{s}$ and adding missing hydrogens to macromolecules. Nucleic Acids Res. 2005;33(Web Server issue):W368-71. doi:10.1093/nar/ gki464.

45. Anandakrishnan $\mathrm{R}$, Aguilar B, Onufriev AV. $\mathrm{H}++$ 3.0: automating $\mathrm{pK}$ prediction and the preparation of biomolecular structures for atomistic molecular modeling and simulations. Nucleic Acids Res. 2012;40(Web Server issue):W537-41. doi:10.1093/nar/gks375.

46. Myers J, Grothaus G, Narayanan S, Onufriev A. A simple clustering algorithm can be accurate enough for use in calculations of pKs in macromolecules. Proteins. 2006;63(4):928-38. doi:10.1002/prot.20922.

47. Onufriev A, Anandakrishnan R, Aguilar B, Gordon J, Myers J, Folta T et al. 2004-2005. http://biophysics.cs.vt.edu/H++. Accessed 22 Jul 2016.

48. Robert X, Gouet P. Deciphering key features in protein structures with the new ENDscript server. Nucleic Acids Res. 2014;42(Web Server issue):W320-4. doi:10.1093/nar/gku316.

49. Fry SC, York WS, Albersheim P, Darvill A, Hayashi T, Joseleau J-P, et al. An unambiguous nomenclature for xyloglucan-derived oligosaccharides. Physiol Plant. 1993;89(1):1-3. doi:10.1111/j.1399-3054.1993.tb01778.x.

\section{Submit your next manuscript to BioMed Central and we will help you at every step:}

- We accept pre-submission inquiries

- Our selector tool helps you to find the most relevant journal

- We provide round the clock customer support

- Convenient online submission

- Thorough peer review

- Inclusion in PubMed and all major indexing services

- Maximum visibility for your research

Submit your manuscript at www.biomedcentral.com/submit
() Biomed Central 